\title{
The omentum of obese girls harbors small adipocytes and browning transcripts
}

\author{
Elena Tarabra, ${ }^{1}$ Jessica Nouws, ${ }^{1}$ Alla Vash-Margita, ${ }^{2}$ Geoffrey S. Nadzam, ${ }^{3}$ Rachel Goldberg, ${ }^{1}$ \\ Michelle Van Name, ${ }^{1}$ Bridget Pierpont, ${ }^{1}$ James R. Knight, ${ }^{4,5}$ Gerald I. Shulman, ${ }^{6,78}$ and Sonia Caprio ${ }^{1}$ \\ Department of Pediatrics, ${ }^{2}$ Department of Obstetrics, Gynecology and Reproductive Sciences, ${ }^{3}$ Department of \\ Gastrointestinal Surgery, and ${ }^{4}$ Department of Genetics, Yale University School of Medicine, New Haven, Connecticut, \\ USA. ${ }^{5}$ Yale Center for Genome Analysis, Yale University West Campus, Orange, Connecticut, USA. 'Department of Internal \\ Medicine, ${ }^{7}$ Department of Cellular and Molecular Physiology, and ${ }^{8}$ Yale Diabetes Research Center, Yale University School of \\ Medicine, New Haven, Connecticut, USA.
}

\begin{abstract}
Severe obesity (SO) affects about $6 \%$ of youth in the United States, augmenting the risks for cardiovascular disease and type 2 diabetes. Herein, we obtained paired omental adipose tissue (omVAT) and abdominal subcutaneous adipose tissue (SAT) biopsies from girls with $\mathrm{SO}$ undergoing sleeve gastrectomy (SC), to test whether differences in cellular and transcriptomic profiles between omVAT and SAT depots affect insulin sensitivity differently. Following weight loss, these analyses were repeated in a subgroup of subjects having a second SAT biopsy. We found that omVAT displayed smaller adipocytes compared with SAT, increased lipolysis through adipose triglyceride lipase phosphorylation, reduced inflammation, and increased expression of browning/beiging markers. Contrary to omVAT, SAT adipocyte diameter correlated with insulin resistance. Following SC, both weight and insulin sensitivity improved markedly in all subjects. SAT adipocytes' size became smaller, showing increased lipolysis through perilipin 1 phosphorylation, decreased inflammation, and increased expression in browning/beiging markers. In summary, in adolescent girls with SO, both omVAT and SAT depots showed distinct cellular and transcriptomic profiles. Following weight loss, the SAT depot changed its cellular morphology and transcriptomic profiles into more favorable ones. These changes in the SAT depot may play a fundamental role in the resolution of insulin resistance.
\end{abstract}

Conflict of interest: The authors have declared that no conflict of interest exists.

Copyright: () 2020, American Society for Clinical Investigation.

Submitted: December 10, 2019 Accepted: February 26, 2020 Published: March 3, 2020.

Reference information: /CI Insight. 2020;5(6):e135448.

https://doi.org/10.1172/jci. insight.135448.

\section{Introduction}

Severe obesity is the fastest-growing subcategory of obesity in youth, afflicting about $6 \%$ of all youth in the United States (1-7). Of note, tracking of adiposity from childhood into adulthood is more pronounced in the severely obese (8-10), with a high body mass index (BMI) in adolescence being associated with increased risk of cardiovascular disease, type 2 diabetes mellitus (T2DM), and premature death (11-16).

Given the limited effectiveness of lifestyle and pharmacological interventions for severe obesity in youth, surgical procedures that have proven health benefits for adults are increasingly being considered for severely obese adolescents $(1,17)$. Recently, Inge et al. provided longitudinal 5-year outcomes of gastric bypass in adolescents as compared with adults, indicating that adolescents had remission of diabetes and hypertension more often than adults (18). The outcomes data from the Teen Longitudinal Assessment of Bariatric Surgery (Teen-LABS) have greatly reinforced the use of bariatric surgery in adolescents with severe obesity (SO), which is now officially recommended by the Endocrine Society Clinical Practice Guideline (19). Despite the clinical relevance of the Teen-LABS studies, the mechanisms by which weight loss induced by bariatric surgery, specifically by sleeve gastrectomy (SG), leads to greater remission in cardiometabolic health in youth is unknown. It is conceivable that changes in the biology of 2 key adipose depots, the omental adipose tissue (omVAT) and subcutaneous abdominal adipose tissue (SAT), after weight loss have a greater modulatory effect on metabolism, given the greater plasticity of white adipose tissue during adolescence. However, the lack of parallel assessment of the omental versus subcutaneous abdominal depots in adolescents with SO greatly limits the understanding of the putative interdepot differences and their potential changes following weight loss after SG.

Lipolysis, the process by which triglycerides (TGs) are hydrolyzed to free fatty acids (FFAs) and glycerol, occurs by sequential action of adipose triglyceride lipase (ATGL), hormone-sensitive lipase (HSL), 
and monoglyceride lipase (MGL) (20-26). The first step of the lipolytic cascade involves the activation/ phosphorylation of ATGL that appears to be dependent on CGI58 association (20-23). During this stage, a lipid droplet-associated surface protein, perilipin 1 (PLIN1), plays an important role during the lipolytic cascade as suppressor of CGI58 (24). When inactivated, PLIN1 binds to CGI58 and blocks the activation of ATGL. When PLIN1 is phosphorylated, it releases CGI58, and therefore the lipolytic cascade can continue. During this first step of lipolysis, triacylglycerol is hydrolyzed to diacylglycerol (DAG) and TG. The second step shows the involvement/phosphorylation of HSL, that uses DAG as substrate and produces monoacylglycerol (MAG) and TG (25). Finally, during the final step of lipolysis, the MGL degrades MAG, generating FFA and glycerol (26). Both hormones/nutrients regulate this important pathway. During negative energy balance states, activation of lipolysis results in a profound increase in FFA release from adipose tissue, thereby providing the organism with substrates for oxidative metabolism.

To dissect differences in cellular morphology and biologic pathways between the omVAT depot compared with those of the SAT, we obtained paired adipose tissues from these 2 depots in a group of obese adolescent girls with SO who were undergoing SG. Additionally, we followed these subjects during their weight loss over a period of a year, and in a subgroup we repeated the abdominal SAT biopsy after weight loss. We tested the following hypotheses: (a) differences in the cellular, lipolytic activity, and transcriptomic profiles of the SAT compared with those of the omVAT depot may play an essential role in the development of insulin resistance in youth with SO; (b) weight loss after the bariatric surgery and the dramatic metabolic changes occurring in the SAT may reduce insulin resistance and its ominous consequences; and (c) weight loss induced by SG might be associated with browning of the SAT depot.

\section{Results}

Anthropometric, clinical, and metabolic characteristics at baseline. From The Yale Study of Body Fat Patterning in Obese Adolescents cohort, we studied 10 female subjects ( 7 adolescents and 3 young adults) with SO (class 2/3 obesity) who underwent bariatric surgery using SG procedure. The anthropometric, clinical, and metabolic characteristics of participants are presented in Table 1. All subjects had a mean age of 18.3 years (range 16-22), a Tanner stage 4 to 5 , and a mean BMI of $45.9 \mathrm{~kg} / \mathrm{m}^{2}$ (range 36.7-57.2). Among all the participants, there were 3 subjects with early T2DM and 2 with impaired glucose tolerance.

Adipocytes' size distribution in SAT and omVAT depots. To determine the difference in adipocytes' morphology, we obtained paired samples from both abdominal SAT and omVAT during the SG surgery and fixed the tissues in osmium tetroxide, as previously described (27).

The adipocyte cell size distribution profile from the SAT depot showed a distinct shift to the right compared with that seen in omVAT, with the adipocytes from SAT being significantly larger than those from omVAT $(P=0.002)$ (Figure 1A and Table 2$)$. Interestingly, while the peak diameter of SAT cells directly correlated with the increase in insulin resistance (HOMA-IR) $(P=0.037)$, in the omVAT such correlation was not present (Figure 1B). Correlations between BMI and cell peak diameter were not statistically significant either in SAT or in omVAT (SAT: $r=-0.21, P=0.56$; omVAT: $r=0.06, P=0.89$ ).

Table 2 shows additional adipose cell size parameters in SAT and omVAT. Interestingly, the percentage of small cells (i.e., cells below nadir) and the ratio of small to large cells in SAT were significantly higher compared with those from the omVAT depots. Of note, we found a strong positive correlation between omVAT cell peak diameter and both percentage of SAT small cells $(r=0.729, P=0.021)$ and SAT ratio of small to large cells $(r=0.754, P=0.015)$ (Figure $1, C$ and D). However, the opposite was not true. These significant correlations suggest a possible cross-talk from omVAT to SAT but not vice versa (SAT to omVAT).

Before SG, omVAT and SAT depots showed similar profiles for genes regulating lipolysis but distinctly different profiles for browning and inflammation markers. To determine potential differential gene signatures/profiles in SAT versus omVAT, we used RNA-sequencing (RNA-Seq). Cluster analysis using the 4155 most significantly expressed genes correctly distinguished SAT and omVAT, as shown by the heatmap (Figure 2A) and principal component analysis (PCA) (Figure 2B). Furthermore, 1492 differentially expressed transcripts were identified (FDR $>0.05$, Padj < 0.05), of which 448 were upregulated $\left(\log _{2}\right.$ fold change $>2$, Padj $<$ $0.05)$ and 1044 were downregulated genes $\left(\log _{2}\right.$ fold change $<-2$, Padj $\left.<0.05\right)$. We elected to focus on genes involved in lipid metabolism, white/browning adipose tissue markers, and inflammation, based on the degree of differential expression of each gene and its known functional roles in metabolic diseases.

Ingenuity Pathway Analysis (IPA) revealed that the top 20 highly enriched canonical pathways were mainly associated with several transcription factor pathways, stem cells' pluripotency, as well as 
Table 1. Clinical and metabolic characteristics of the obese adolescents undergoing bariatric surgery

\begin{tabular}{|c|c|c|}
\hline & Mean & Range \\
\hline Age (years) & 18.3 & $16-22$ \\
\hline Ethnicity (nH/H) & $4 / 6$ & \\
\hline Tanner stage (4/5) & $5 / 5$ & \\
\hline \multicolumn{3}{|l|}{ Anthropometrics } \\
\hline Weight (kg) & 125.7 & $96.9-178.7$ \\
\hline Height (cm) & 165.1 & $155-185.4$ \\
\hline $\mathrm{BMI}\left(\mathrm{kg} / \mathrm{m}^{2}\right)$ & 45.9 & $36.7-57.2$ \\
\hline$\%$ fat mass & 48.8 & $42.8-56.6$ \\
\hline Class of obesity $(2 / 3)$ & $1 / 9$ & \\
\hline \multicolumn{3}{|l|}{ Metabolic measurements } \\
\hline Fasting glucose (mg/dL) & 87.3 & $69-100$ \\
\hline Two-hour glucose (mg/dL) & 130.4 & $80-202$ \\
\hline Fasting insulin ( $\mu \mathrm{U} / \mathrm{mL})$ & 33.13 & $9.4-71$ \\
\hline HOMA-IR & 6.99 & $0.5-17.51$ \\
\hline Leptin (ng/mL) & 127.21 & $84.38-223.66$ \\
\hline \multicolumn{3}{|l|}{ Lipids } \\
\hline Total cholesterol (mg/dL) & 167.9 & $121-261$ \\
\hline HDL (mg/dL) & 45 & $27-57$ \\
\hline LDL (mg/dL) & 101.6 & $61-190$ \\
\hline TGs (mg/dL) & 113.4 & $40-256$ \\
\hline FFAs $(\mu \mathrm{mol} / \mathrm{L})$ & 0.574 & $0.338-0.765$ \\
\hline \multicolumn{3}{|l|}{ Liver } \\
\hline $\operatorname{ALT}(\mathrm{U} / \mathrm{L})$ & 32.3 & $14-96$ \\
\hline AST (U/L) & 23.5 & $17-42$ \\
\hline
\end{tabular}

Demographic, anthropometric, and metabolic characteristics are shown for 10 obese adolescent females undergoing gastric sleeve bariatric surgery. $\mathrm{nH}$, non-Hispanics; H, Hispanics; HOMA-IR, Homeostatic Model Assessment of Insulin Resistance; ALT, alanine aminotransferase; AST, aspartate aminotransferase.

WNT/ $\beta$-catenin signaling and immune cell (both granulocyte and agranulocyte) migration. Surprisingly, IPA pointed out a significant differential regulation of genes associated with the browning of white adipose tissue in SAT compared with omVAT. In particular among all the genes associated with this process, $14 \%$ were downregulated and 5\% upregulated in SAT (Figure 2C).

Using RNA-Seq analysis we found a distinct pattern in gene expression between SAT and omVAT (Figure 2, A and B). Surprisingly, we did not observe any significant difference in the expression of the majority of the key genes involved in lipogenesis and lipolysis (Supplemental Figure 1, A-C; supplemental material available online with this article; https://doi.org/10.1172/jci.insight.135448DS1), as well as many of the genes involved in adipogenesis (Supplemental Figure 1E). However, we found a significant increase in leptin $(L E P)$, CCAAT enhancer binding protein- $\beta$, and phosphoenolpyruvate carboxykinase 2 (PCK2) gene expression (Supplemental Figure 1, D and E). Of particular note, we found in omVAT a significantly increased expression in genes associated with the browning of white adipose tissue (28-33) (including $P G C 1 A, U C P 1, C I D E A$, and EBF2) compared with the SAT depot (Supplemental Figure 1, F and G). Interestingly, $C D 36$ (described as a lipid intake channel and positively correlated with adipocytes' differentiation) was also significantly reduced in omVAT compared with the SAT depot (Supplemental Figure 1H).

In addition, we studied potential differential expression of genes regulating inflammation in omVAT and SAT. Overall, omVAT did display a slight reduction in macrophage content by $C D 68$ gene expression and a consistently significant decrease in several proinflammatory genes (including $I L 6, M C P 1$, iNOS, IL8, CD11C) compared with SAT. However, the profile of antiinflammatory genes (Supplemental Figure 1I) was not significantly different between the depots.

To quantify more precisely the differential expression found by RNA-Seq analysis, the expression levels of genes involved in lipid metabolism, browning, and inflammation were confirmed and validated by realtime PCR. As already shown by RNA-Seq, we did not find significant differences by real-time PCR between SAT and omVAT in the expression of the major lipolytic, lipogenic, and adipogenic genes (Figure 3, A-C). 

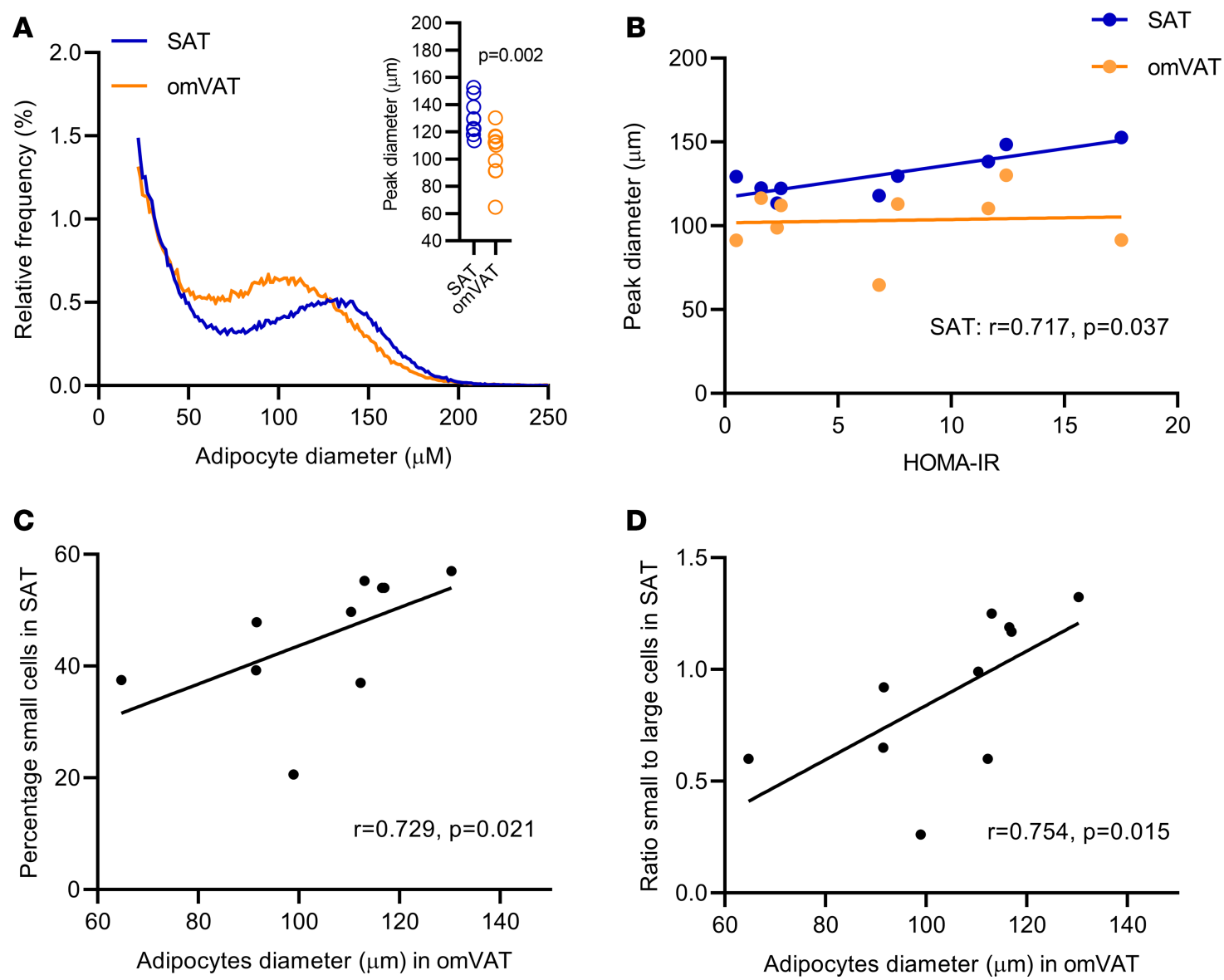

Figure 1. Multisizer adipose cell profile from SAT and omVAT depots. (A) Multisizer cell profiles for SAT and omVAT depots collected from 10 girls with SO undergoing SG using the mean parameters from the curve-fitting formula and adipocyte peak diameter (in inset). (B) Correlation between HOMA-IR and SAT or omVAT cell peak diameter $(n=9)$. (C) Strong correlation between omVAT adipocytes' peak diameter and percentage of small cells in SAT $(n=10)$. (D) Strong direct correlation between omVAT adipocytes' peak diameter and ratio of small to large cells in SAT ( $n=10)$. Cell peak diameter data were compared using Wilcoxon's matched-pairs signed-rank test, and correlations were analyzed using nonparametric Spearman's test. $P$ values less than 0.05 were considered significant.

Only LEP and CD36 were significantly reduced in omVAT compared with SAT (Figure 3, B and C). Interestingly, we observed a slight reduction of CD68 gene expression (a general inflammation marker) as well as of several proinflammatory markers (including IL6, MCP1, and TNFA) in omVAT compared with SAT, but no differences were observed among the antiinflammatory markers (Figure 3D). Using a specific antibody against CD68, we were also able to visualize and localized by IHC the macrophages in both omVAT and SAT $($ SAT CD68 $=22.0 \pm 4.0$ macrophages $/ 100$ adipocytes; omVAT CD68 $=14.9 \pm 2.9$ macrophages $/ 100$ adipocytes; $P=0.09$ ). These representative images showed a slight reduction of $\mathrm{CD}^{+} 8^{+}$cells in omVAT compared with SAT depot (Figure 3E and Supplemental Figure 2A).

Because we found by both IPA and RNA-Seq analysis an increased expression in mitochondrial markers and genes associated with the browning of adipose tissue, we focused our attention on these genes. Using real-time PCR we were able to confirm the increase in gene expression of several genes associated with browning of white adipose tissue (including UCP1, PGC1A,CIDEA, and EBF2) and specific mitochondrial genes (including COX4L1, COX7C, PCK2, and creatine kinase 1 [CKMT1]) in the omVAT depot (Figure 4A).

Given that the browning/beiging of white adipose tissue in both human and mouse models is modulated by cold exposure and/or winter (34-39), we tested in our subjects whether the season could affect the expression of browning/beiging markers in SAT. Overall, we did not observe any significant increase in browning/beiging gene expression in SAT from patients who underwent biopsy during the cold season (winter/fall) compared with those who underwent SG during the warm season (summer/spring) (5 subjects each group, data not shown). These observations are in line with the observation previously published by Kern et al. in SAT from an obese population (40). 
Table 2. Comparison of adipose cell size variables in SAT and omVAT depots from all patients $(n=10)$

\begin{tabular}{lccc}
\hline Cell size variables & SAT & omVAT & P value \\
Peak diameter $(\mu \mathrm{m})$ & $129.73 \pm 4.12$ & $104.60 \pm 5.85$ & $\mathbf{0 . 0 0 2}$ \\
Fraction of large cells & $0.68 \pm 0.07$ & $0.96 \pm 0.10$ & $\mathbf{0 . 0 0 2}$ \\
Nadir $(\mu \mathrm{m})$ & $79.61 \pm 3.35$ & $61.06 \pm 4.04$ & $\mathbf{0 . 0 0 2}$ \\
Ratio small/large cells & $0.90 \pm 0.11$ & $0.56 \pm 0.10$ & $\mathbf{0 . 0 0 6}$ \\
Percentage of small cells & $45.23 \pm 3.64$ & $33.38 \pm 4.45$ & $\mathbf{0 . 0 1}$ \\
Median $(\mu \mathrm{m})$ & $85.19 \pm 4.69$ & $83.13 \pm 3.67$ & 0.84 \\
Mean $(\mu \mathrm{m})$ & $88.21 \pm 2.47$ & $83.40 \pm 2.90$ & 0.23
\end{tabular}

Data are expressed as mean \pm SEM. The nonparametric 2-tailed paired Student's $t$ test was used for comparing SAT versus omVAT, and $P$ values less than 0.05 were considered significant (in bold).

Of note, omVAT adipocyte cell peak diameter showed a strong inverse correlation with the gene expression of UCP1 (master regulator of the browning/beiging markers), PGC1A, and EBF2 (Figure 4, $\mathrm{D}-\mathrm{F})$. Similar correlations were also observed with the mitochondrial gene expression of PCK2, CKMT1, and COX4L1 (Figure 4, E-G). Interestingly, several other genes that failed to achieve the statistically significant difference between SAT and omVAT depots did show a strong and significant inverse correlation between gene expression and adipocyte peak in omVAT (CITED1: $r=-0.770, P=0.013 ;$ TBX1: $r=-0.867$, $P=0.002$; CKMT2: $r=-0.758, P=0.015$; COX7C: $r=-0.634, P=0.054)$. Regardless, no correlation was observed between SAT adipocytes' peak and gene expression (data not shown). These data indicate that in the omVAT depot the browning of white tissue is associated with adipocytes with small diameter.

Given that we observed a significant induction in browning of omVAT compared with SAT, we investigated in paired SAT and omVAT from different subjects the expression at the protein level of UCP1, the major browning/beiging marker. As shown in Figure $4 \mathrm{H}$ and Supplemental Figure 2, B and C, by immunofluorescence, UCP1 staining was strongly increased in the omVAT compared with SAT depot (SAT fluorescence intensity = $9.2 \pm 1.5 \mathrm{AU}$; omVAT fluorescence intensity=19.8 $\pm 1.2 \mathrm{AU} ; P=0.002)$, supporting the real-time PCR data.

ATGL but not HSL phosphorylation regulates omVAT adipocytes' cell size, enhancing lipolysis. Because omVAT adipocytes showed smaller cell peak diameter compared with SAT adipocytes, but RNA-Seq profile data of lipid metabolism regulatory genes were not able to explain this phenomenon, we examined whether differences in the function/activity at the protein level of 2 of the master regulators in lipolysis, ATGL and HSL, would emerge between the 2 depots and whether the phosphorylation of these key proteins would be correlated with adipocyte size. Therefore, we compared the protein expression level of phosphorylated (p-) ATGL and p-HSL in SAT and omVAT. In particular, we observed a significant increase in p-ATGL in omVAT compared with SAT $(P<0.01)$ and a trend of increasing HSL phosphorylation (Figure 5, A-C). Moreover, surprisingly, p-ATGL significantly correlated with SAT cell diameter $(r=0.742, P=0.042)$ but not omVAT cell diameter (Figure 5D), and notably, p-ATGL correlated with HOMA-IR in both SAT and omVAT (Figure 5E). No significant correlations were observed between p-HSL and cells' peak diameter or HOMA-IR in both omVAT and SAT (data not shown).

Interestingly, in obese adolescents PLIN1 protein expression was undetectable in both the SAT and omVAT depot (data not shown).

Effects of SG on changes in weight, insulin sensitivity, and fatty liver. After bariatric surgery only 1 patient discontinued the follow-up for personal reasons and therefore was not included in the analysis. As expected, all patients who underwent SG had a marked reduction in body weight, absolute BMI, and percentage change in BMI (Table 3), reaching after 6 months a plateau in body weight loss, which was maintained through the 10-month follow-up visit (Figure 6A). Interestingly, 6 out of 9 patients had a repeated measure of insulin sensitivity using the HOMA-IR, which indicated a strong improvement in insulin sensitivity, particularly in the obese girls who were more insulin resistant (Figure 6B).

During the follow-up period, 4 girls consented to having a second abdominal SAT biopsy, thus providing the unique opportunity to compare the changes in the SAT depot characteristic before (baseline) and after weight loss (follow-up biopsy). These 4 subjects were representative of the main group because they showed a reduction in BMI, percentage change in BMI, and body weight after surgery similar to the other subjects (Table 3 and Supplemental Figure 3, A-D). 
A

Significant genes in SAT vs omVAT

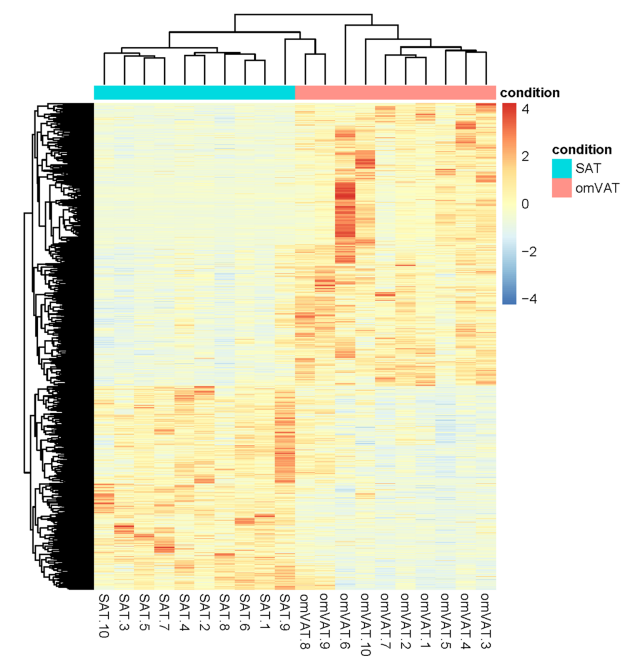

B

PCA plot (significant genes, TPM values)

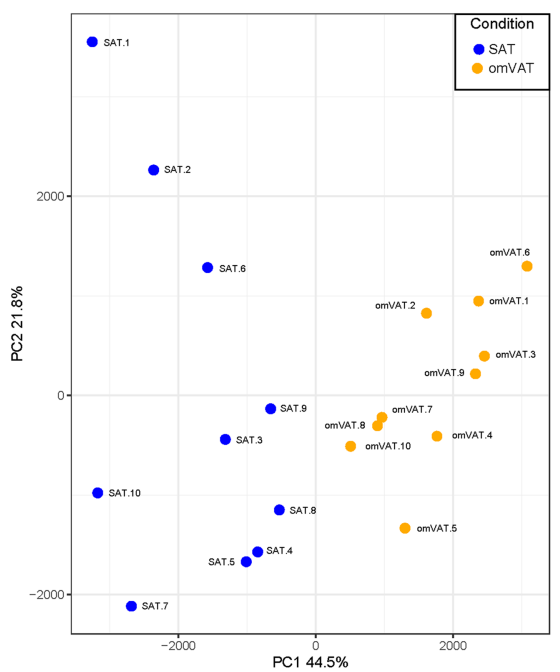

C

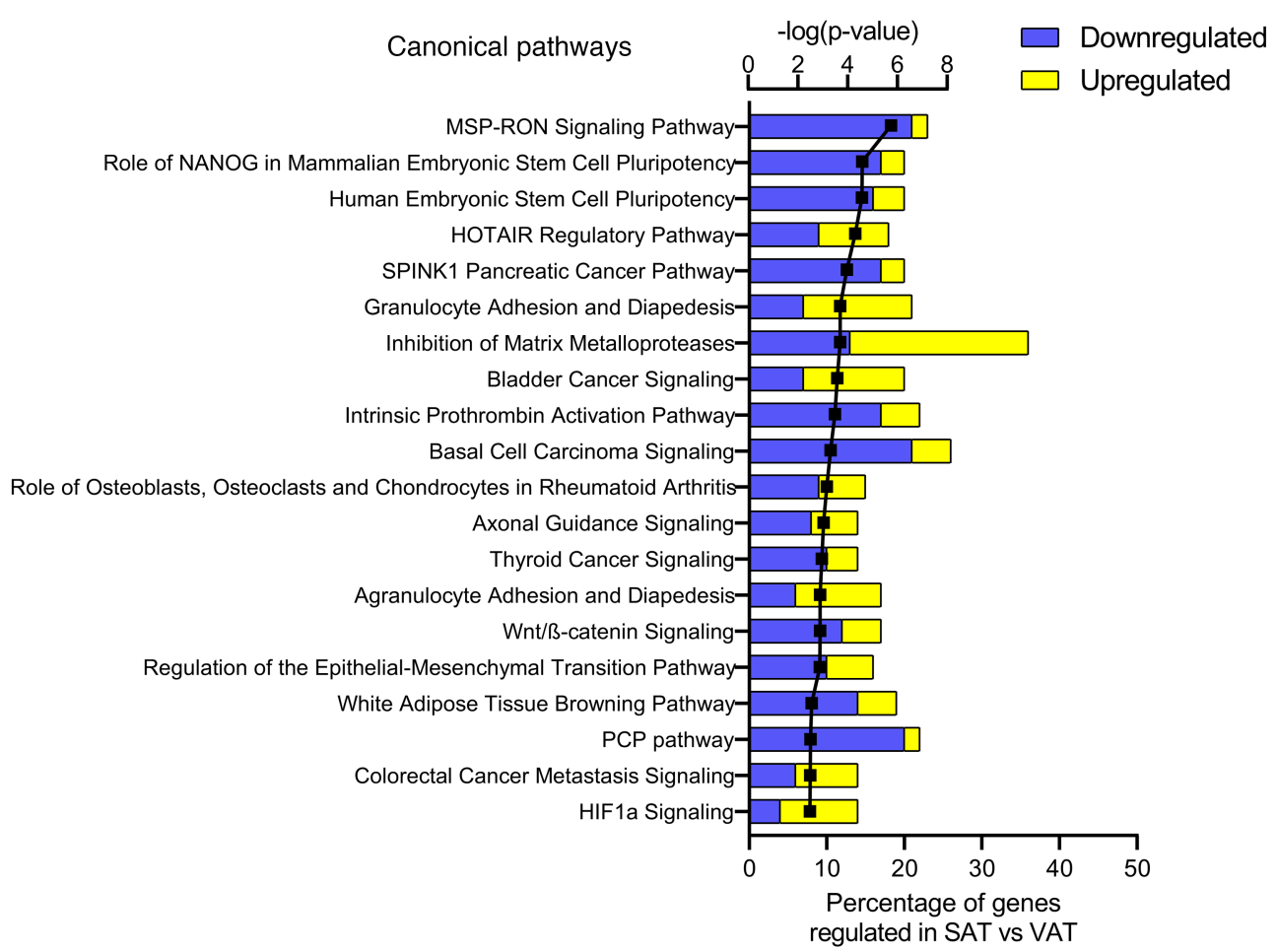

Figure 2. Transcriptome analysis in SAT and omVAT. (A) RNA sequencing (RNA-Seq) analysis of SAT and omVAT with heatmap and clustering of the 4155 most variably expressed genes in the RNA-Seq data set. (B) PCA analysis of the significant genes. (C) Canonical pathways enrichment analysis for upregulated (in yellow) and downregulated (in blue) genes between SAT and omVAT samples. The bar represents the percentage of genes up-/downregulated within the specific canonical pathways. The solid line represents the $-\log (P$ value $)$ for each pathway. Analysis of $n=10$ samples each depot.

SAT adipocytes are smaller after weight loss induced by $S G$. The Multisizer adipose cell size profile from the SAT follow-up biopsy showed a clear shift to the left compared with the baseline biopsy cell curve profile. The cell peak calculated for the baseline biopsy $(124.86 \pm 5.61 \mu \mathrm{m})$ was significantly higher than the cell peak calculated from the follow-up biopsy $(99.99 \pm 0.71 \mu \mathrm{m}, P=0.0046)$ (Figure 6, C and D, and Supplemental Figure 3, E-H).

Gene profiling after weight loss showed an increase in lipolytic genes, and induction of genes regulating browning activity, but a reduction in proinflammatory genes in the SAT depot. We analyzed using both RNA-Seq and 
A

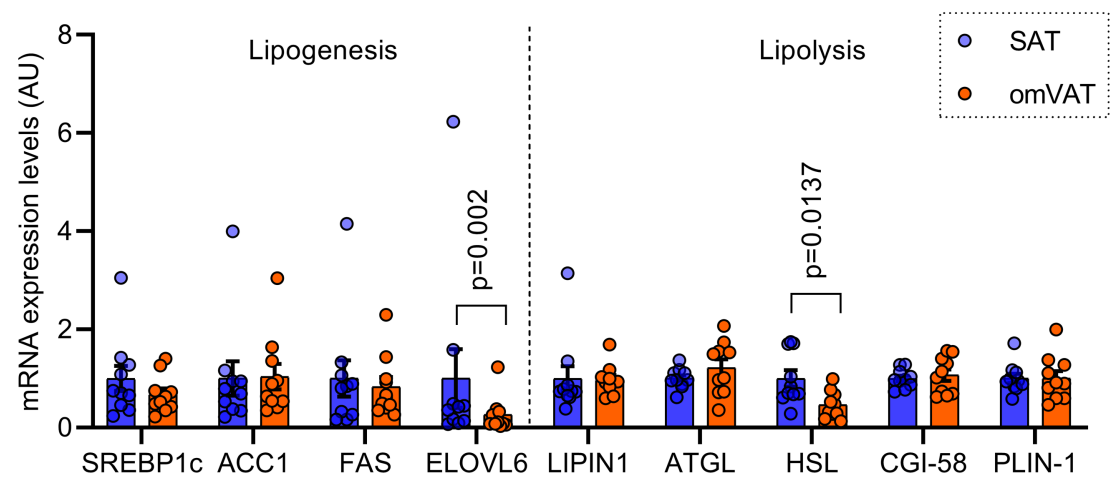

B

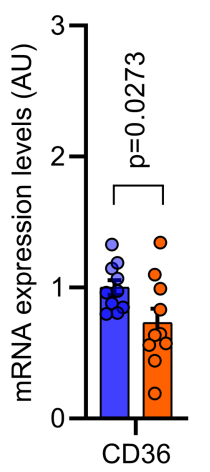

C $\quad 47 \quad$ Adipogenesis

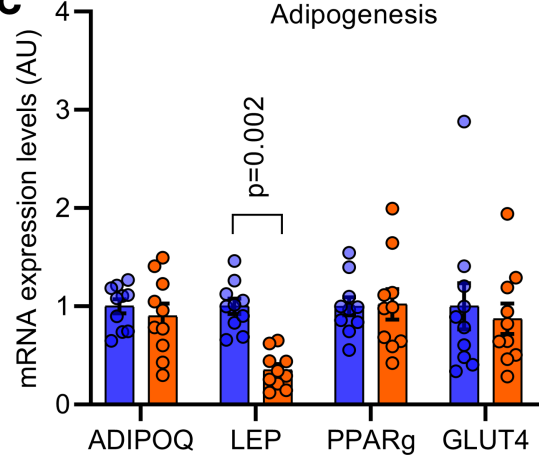

D
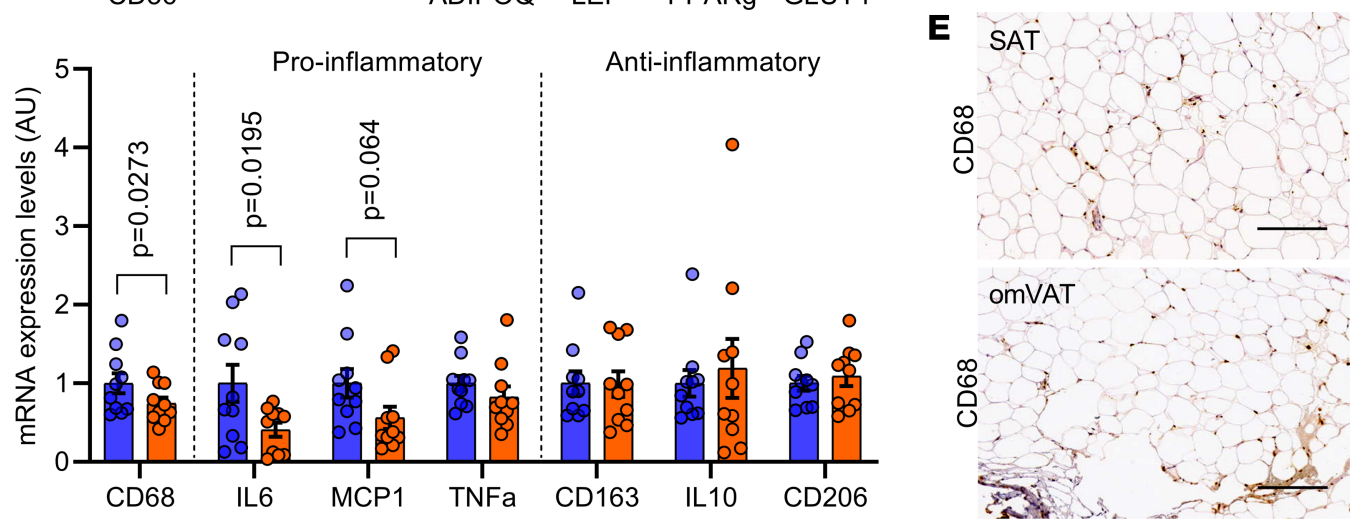

Figure 3. Evaluation by real-time PCR of a panel of genes associated with lipid metabolism, adipogenesis, browning of white adipose tissue, and inflammation in SAT and omVAT. Validation by real-time PCR of RNA-Seq results of a panel of genes involved in (A) lipid metabolism, (B and C) adipogenesis, and $(\mathbf{D})$ inflammation $(n=10)$. (E) Representative images of $C D 68^{+}$macrophage IHC staining showing a slight reduction in macrophage infiltration in omVAT compared with SAT. Scale bar: $200 \mu \mathrm{m}$. Error bars represent mean \pm SEM. Gene expression data were compared using Wilcoxon's matched-pairs signed-rank test. $P$ values less than 0.05 were considered significant.

real-time PCR the expression of the same panel of genes associated with lipid metabolism, browning, and inflammation markers in SAT obtained at baseline and at follow-up biopsy from the same subjects. We found an increase in lipolytic and adipogenic genes' (including ATGL, HSL, PLIN1, PLIN4, and ADIPOQ) expression levels in the follow-up biopsy compared with the baseline biopsy (Figure 7, A-C, and Supplemental Figure 4, A-C).

In contrast, we found a reduction in the proinflammatory status of SAT after weight loss as shown by stark downregulation of $I L 6, M C P 1, I L 8, I L 1 B$, and $C D 11 C$ (Figure 7D and Supplemental Figure 4D). Overall, the total amount of macrophages was maintained between baseline and follow-up biopsy, as shown by CD68 mRNA expression levels (Figure 7D) and IHC using a specific CD68 antibody (baseline biopsy CD68 $=22.0 \pm 4.0$ macrophages $/ 100$ adipocytes; follow-up biopsy CD68 $=18.3 \pm 2.4$ macrophages $/ 100$ adipocytes; $P=0.493$ ) (Figure 7E and Supplemental Figure 5A).

Of note, an increase in genes that regulate the browning of SAT was also observed after weight loss as well as genes associated with mitochondrial activity (Figure 8, A and B, and Supplemental Figure 4, E and F). 
A

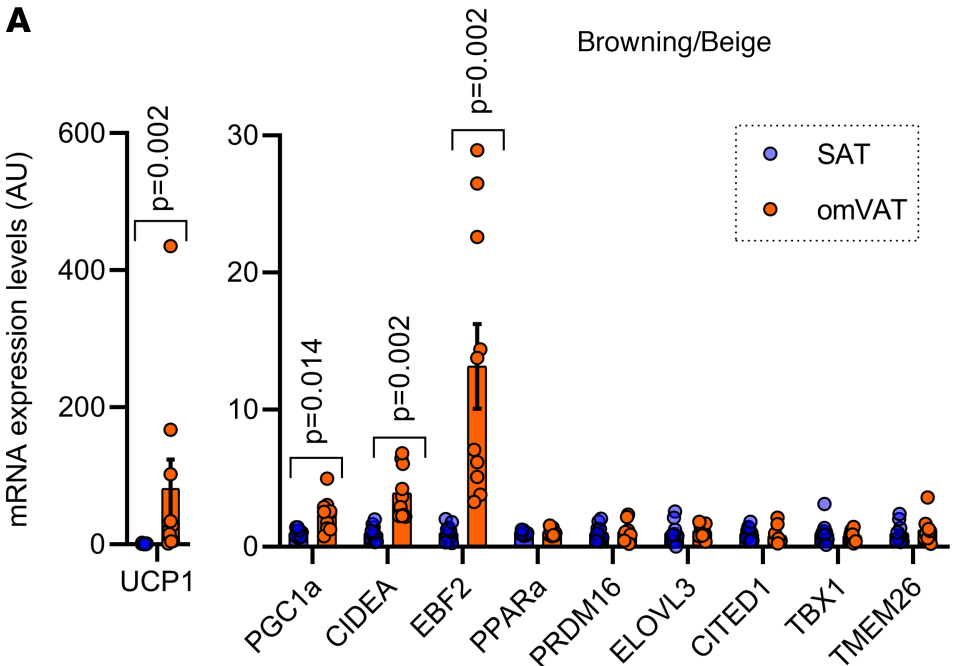

Mitochondria

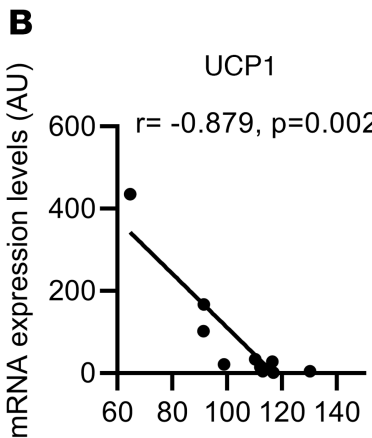

C

PGC1a

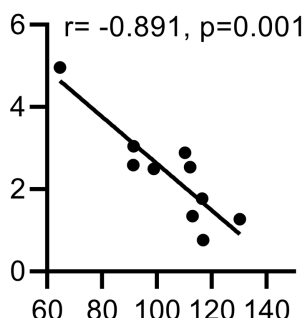

omVAT adipocytes peak diameter $(\mu \mathrm{m})$
$\mathbf{F}$

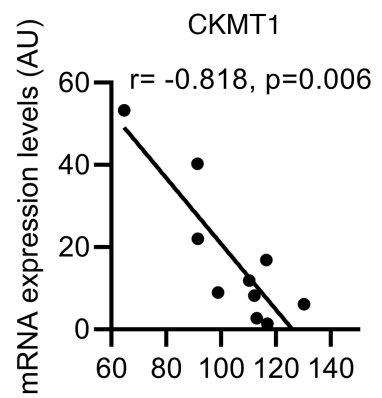

G

COX4L1

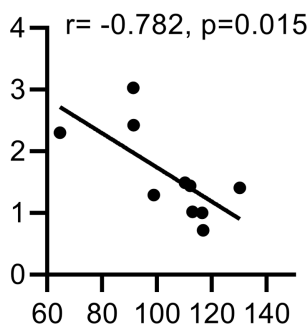

omVAT adipocytes peak diameter $(\mu \mathrm{m})$

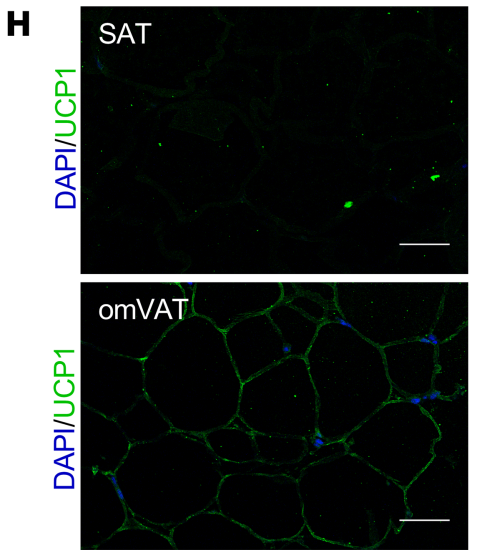

Figure 4. Distinct profile for browning markers in omVAT compared with SAT depots. Validation by real-time PCR of RNA-Seq results of a panel of genes involved in (A) browning of white adipose tissue. Strong inverse correlation between omVAT adipocyte peak diameter and (B-D) browning gene expression or $(\mathbf{E}-\mathbf{C})$ mitochondrial markers $(n=10)$. (H) Representative immunofluorescence staining images showing an increase of UCP1 in omVAT compared with SAT. Scale bar: $50 \mu \mathrm{m}$. Error bars represent mean \pm SEM. Gene expression data were compared using Wilcoxon's matchedpairs signed-rank test, and correlations were analyzed using nonparametric Spearman's test. $P$ values less than 0.05 were considered significant.

The increase of CIDEA gene expression was also assessed by immunofluorescence (Figure 8C and Supplemental Figure 5, B and C). The specific staining for CIDEA supported the mRNA expression results and showed a strong increase in SAT tissue obtained from a biopsy after weight loss (CIDEA fluorescence intensity $=40.7 \pm 2.6 \mathrm{AU}$ ) compared with the staining from the baseline biopsy (CIDEA fluorescence intensity $=26.8 \pm 2.6 \mathrm{AU} ; P$ value baseline vs. after weight loss $=0.0098)$. It should be noted that all the follow-up biopsies were done during the warm season (spring/summer), which would exclude the possibility that the observed browning/beiging of the white adipose tissue was due to any seasonal effects. 
A

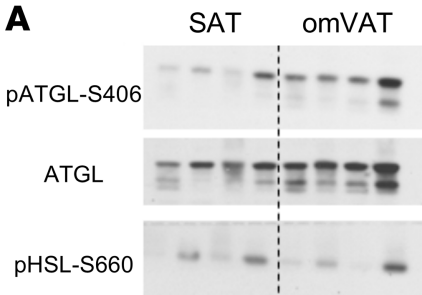

HSL

Actin

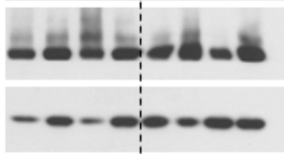

B

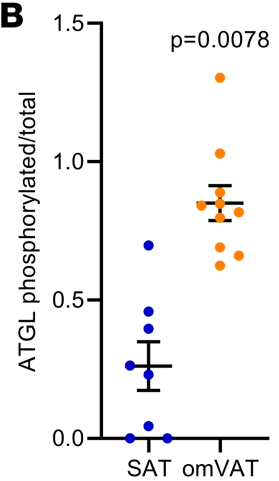

$\mathbf{E}$

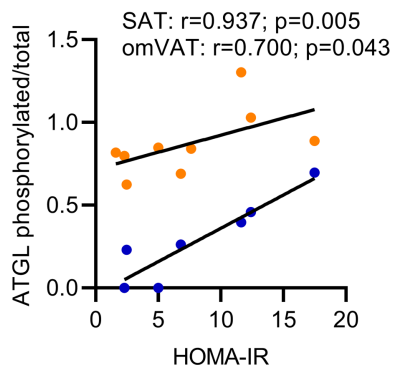

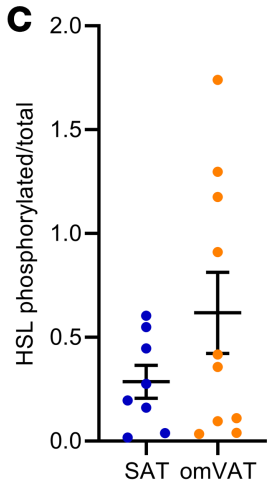

- SAT

- omVAT

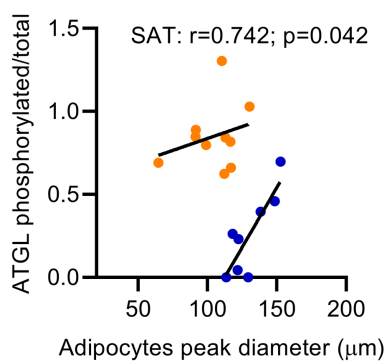

Figure 5. Increase in lipolytic activity in omVAT mainly through ATCL phosphorylation. (A) Representative blots showing ATCL and HSL phosphorylation in SAT $(n=8)$ and omVAT $(n=10)$. (B and C) Quantification of the phosphorylated/total ratio both of ATCL and HSL. (D) Positive correlation of ATCL phosphorylation with adipocytes' peak diameter in SAT but not omVAT. (E) Positive correlation of ATGL phosphorylation with HOMA-IR in SAT ( $n=$ 8) and omVAT $(n=9)$. Error bars represent mean \pm SEM. Protein phosphorylation was analyzed using Wilcoxon's matched-pairs signed-rank test, and correlations were analyzed using nonparametric Spearman's test. $P$ values less than 0.05 were considered significant.

PLIN1 but not ATGL phosphorylation regulates SAT adipocytes' cell size after weight loss. Because we found that the posttranslational activation of the lipolytic proteins is the key indicator of the lipolytic activity in adipocytes, we investigated the level of p-ATGL and p-PLIN1 in SAT samples obtained at baseline during bariatric surgery and follow-up biopsy (Figure 9, A and B). Surprisingly, we observed that after weight loss, there was a slight reduction in the ATGL phosphorylation. On the other hand, this depot showed a strong and significant overexpression of the total form of PLIN1 (which was almost not expressed in SAT before weight loss) (Figure 9C), and consequently we were able to detect the phosphorylation of PLIN1 after weight loss. These data indicate that during the weight loss the reduction of adipocyte size and therefore adipocyte lipolytic activity may be mainly under the control of PLIN1.

\section{Discussion}

The present study provides insights into the potential underlying differences in the cellular and transcriptomic profiles of paired biopsy samples from the omental (omVAT) and subcutaneous abdominal (SAT) depots, from a group of adolescent girls with SO, with a spectrum of insulin sensitivity, undergoing SG. Following SG, weight changes were closely monitored in the entire group. In a subset of subjects, a repeated SAT biopsy was performed and measurements of cellularity and gene expression were repeated, during weight stabilization.

The major findings, summarized in Figure 10, are as follows.

First, at baseline, before SG, we found that omVAT compared with SAT displayed (a) smaller adipocytes and increased lipolytic activity, (b) reduction of proinflammatory markers, (c) increased markers of browning/beiging of white adipose tissue, and (d) (opposite from SAT) adipocytes' peak diameter did not correlate positively with insulin sensitivity.

Second, after SG-induced weight loss, all subjects showed (a) a marked reduction in body weight, absolute BMI, and percentage change in BMI, reaching after 6 months a plateau in body weight loss, which 
Table 3. Comparison of anthropometric characteristics between all follow-up and selected returning patients

\begin{tabular}{|c|c|c|c|c|c|c|c|c|c|c|c|c|}
\hline & \multicolumn{6}{|c|}{ All follow-up patients $(n=9)$} & \multicolumn{6}{|c|}{ Returning girls ( $n=4$ ) } \\
\hline & $\begin{array}{c}\text { Baseline } \\
(n=9)\end{array}$ & After 6 mo & $\begin{array}{c}P \text { value } \\
\text { vs. } \\
\text { baseline }\end{array}$ & $\begin{array}{l}\text { After } 10 \\
\text { mo }\end{array}$ & $\begin{array}{c}P \text { value } \\
\text { vs. } \\
\text { baseline }\end{array}$ & $\begin{array}{c}P \text { value } \\
6 \text { mo vs. } \\
10 \text { mo }\end{array}$ & After 6 mo & $\begin{array}{c}P \text { value } \\
\text { vs. } \\
\text { baseline }\end{array}$ & $\begin{array}{c}P \text { value } \\
\text { vs. } 6 \mathrm{mo}^{\mathrm{A}}\end{array}$ & $\begin{array}{l}\text { After } 10 \\
\text { mo }\end{array}$ & $\begin{array}{c}P \text { value } \\
\text { vs. } \\
\text { baseline }\end{array}$ & $\begin{array}{c}P \text { value } \\
\text { vs. } 10 \mathrm{mo}^{\mathrm{A}}\end{array}$ \\
\hline $\mathrm{HT}(\mathrm{cm})$ & $163.0 \pm 1.6$ & $164.4 \pm 1.7$ & 0.016 & $164.6 \pm 1.8$ & 0.016 & ns & $162.5 \pm 2.1$ & ns & ns & $162.7 \pm 2.1$ & ns & ns \\
\hline $\mathrm{BW}(\mathrm{kg})$ & $120.9 \pm 6.4$ & $95.3 \pm 5.5$ & 0.004 & $96.8 \pm 5.9$ & 0.004 & ns & $88.2 \pm 7.2$ & ns & ns & $87.3 \pm 7.0$ & ns & ns \\
\hline $\mathrm{BMI}$ & $45.7 \pm 2.3$ & $35.4 \pm 2.2$ & 0.004 & $36.1 \pm 2.3$ & 0.004 & ns & $33.6 \pm 3.0$ & ns & ns & $33.4 \pm 2.8$ & ns & ns \\
\hline$\%$ fat & $48.8 \pm 1.4$ & $40.3 \pm 1.7$ & 0.016 & $39.6 \pm 1.8$ & 0.06 & ns & $40.4 \pm 2.8$ & ns & ns & $40.3 \pm 2.1$ & ns & ns \\
\hline \multicolumn{13}{|c|}{ Change from baseline } \\
\hline BW (\%) & & $-21.3 \pm 1.2$ & 0.004 & $-20.0 \pm 2.5$ & 0.004 & ns & $-20.7 \pm 2.5$ & ns & ns & $-21.5 \pm 2.9$ & ns & ns \\
\hline BMI (\%) & & $-22.8 \pm 1.5$ & 0.004 & $-21.3 \pm 2.5$ & 0.004 & ns & $-22.5 \pm 3.0$ & ns & ns & $-22.8 \pm 3.2$ & ns & ns \\
\hline
\end{tabular}

All values are expressed as mean \pm SEM. The nonparametric 2 -tailed paired Student's $t$ test was used for comparing groups. $P$ values less than 0.05 were considered significant. ${ }^{A} P$ value of the returning 4 girls compared with all girls at the same follow-up time. BW, body weight; HT, height.

was maintained through the 10-month follow-up visit; and (b) a marked improvement in insulin sensitivity, particularly in the obese girls who were more insulin resistant.

Third, in the 4 subjects who agreed to have a repeated SAT biopsy after weight loss, we found (a) reduction in cells' diameter compared with the baseline biopsy and improvement in insulin sensitivity, (b) increased lipolytic activity, (c) strong induction of browning markers, and (d) and a substantial reduction of proinflammatory markers.

A unique aspect of this study is the particularly young age of the subjects, who were studied during adolescence, a developmental and maturational stage during which the adipose tissue experiences not only changes in body fat distribution but also a great accretion in fat mass. Indeed, adolescence is considered a critical time for obesity development (41). Given the major sex differences in body fat distribution (42-45) and higher prevalence rates of SO among females, we elected to study only girls.

There is very limited information regarding the differences in the cellularity and transcriptomic profiles of the omental and SAT depots from obese adolescents with SO. Likely due to the greater plasticity of the white adipose tissue at this particular young age (46-48), the findings in the cellularity, the transcriptomic profiles, and their responses to SG-induced weight loss may be specific to this developmental stage of adolescence, compared with those described in older subjects with obesity.

Interdepot differences in cell size. Adipocytes from the omental depot (omVAT) were smaller compared with those from the SAT. These cell size differences are consistent with those reported in adult obese women (49). Surprisingly, we found no relation between cell size and insulin sensitivity in the omVAT in contrast to the significant correlation with the large cell diameter and insulin sensitivity in the SAT. This finding would support the primary role of the SAT rather than the omVAT in the development of insulin resistance at this particular developmental stage.

Further analysis of the cellular patterns suggests that in adolescents with SO, the proportion of small/large cells and the percentage of small cells from the SAT depot were significantly higher compared with those seen from the omVAT depot (see Table 2). Of note, the cell diameter of the adipocytes from the omVAT depot positively correlated with the ratio of small to large cells from the SAT ( $r=0.754, P=0.015$, Figure $1 \mathrm{D})$. The reason for the differential small cell accumulation between the 2 depots is not clear but could be related to the regional differences of fat tissue and presence of insulin resistance. To some extent, our results regarding the interdepot differences in the proportion of small/large cells are different from those reported in adults by Liu et al. This discrepancy is likely due to the difference in the age of the subjects studied, that is, adolescents versus adults (49).

To the best of our knowledge, our study may be the first to document, using Multisizer technology, that increasing cell size in omVAT is associated with accumulation of small cells in SAT in adolescent girls with SO. Additionally, it suggests that the cell diameter of the adipocytes from the omVAT depot may regulate the proportion of the small to large cells in the SAT (see Figure 1). Regardless of the directionality of these relations, what is emerging is that the presence of large cells and the greater proportion of small to large cells in the SAT may be playing a greater role in shaping the onset of insulin resistance compared with that seen in the omVAT. Whether this difference is due to the particular maturational stage or due to the regional site of the depot remains unclear. 
A

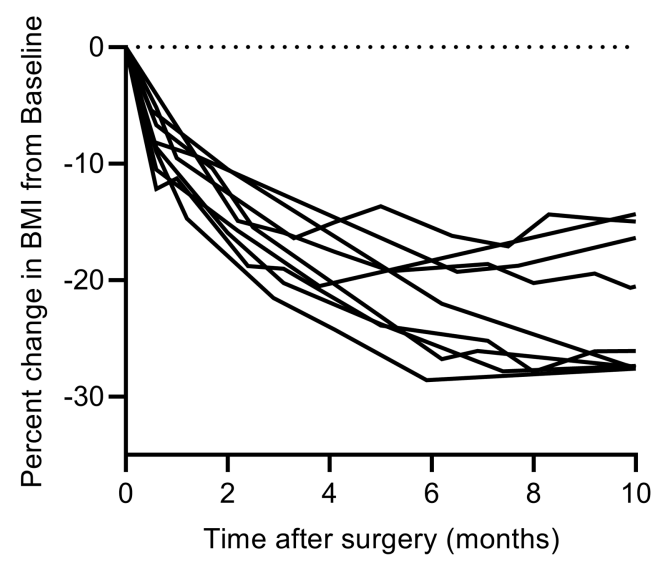

C

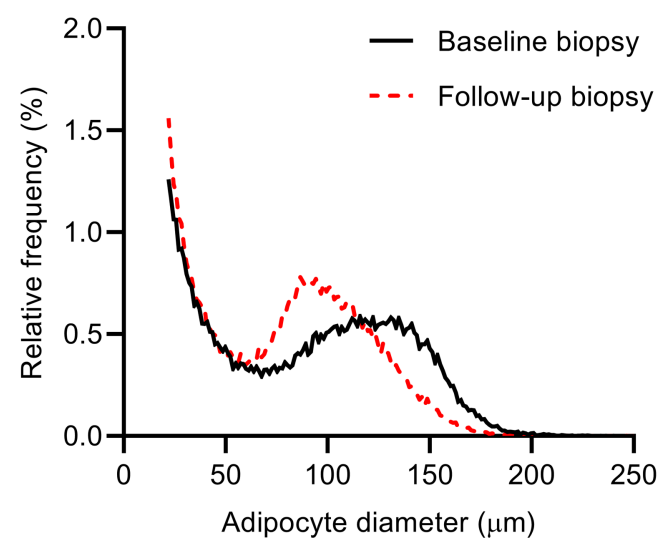

B

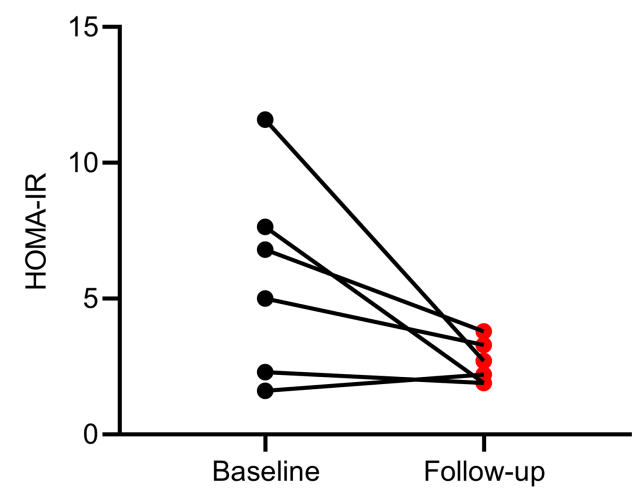

D

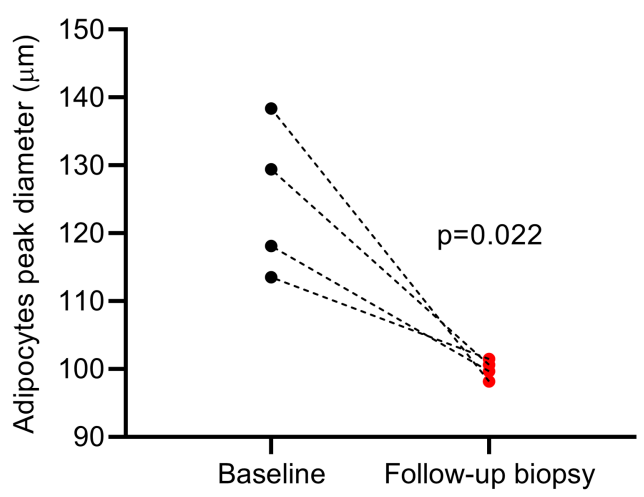

Figure 6. Reduction in body weight, improvement in insulin sensitivity, and changes in adipocyte diameter after weight loss. (A) Reduction in body weight as percentage change in BMI from baseline (dotted line). Each solid line represents an individual participant $(n=9)$ during the follow-up visits. (B) Improvement in insulin sensitivity after weight loss $(n=6)$. (C) Multisizer cell profiles in SAT as mean of 4 subjects at the surgery time and returning for a second SAT biopsy after weight loss. (D) Adipocyte peak diameter from SAT at baseline biopsy and at the follow-up biopsy after weight loss $(n=4)$. Data were compared using Wilcoxon's matched-pairs signed-rank test. $P$ values less than 0.05 were considered significant.

To further understand the interdepot differences in cellularity, we examined whether differences in the function/activity at the protein level of 2 of the master regulators in lipolysis, ATGL and HSL, would emerge between the 2 depots and whether the phosphorylation of these key proteins would be correlated with adipocyte size. To this end, we compared the protein expression level of p-ATGL and p-HSL in SAT and omVAT. In particular, we found a significant increase in p-ATGL in omVAT compared with SAT $(P<0.01)$ and a trend of increasing HSL phosphorylation, which did not reach statistical significance (Figure 5, A-C). Surprisingly, in SAT p-ATGL levels significantly correlated with cell diameter $(r$ $=0.742, P=0.042$ ) but not in omVAT (Figure 5D), and notably, both SAT and omVAT p-ATGL strongly correlated with HOMA-IR (Figure 5E). No significant correlations were observed between p-HSL and cells' peak diameter or HOMA-IR in both omVAT and SAT (data not shown). Although phosphorylation of ATGL would suggest greater lipolytic activity of omVAT, the fact that p-ATGL significantly correlated with SAT cell size diameter and HOMA-IR supports the idea of SAT having a primary role in the development of insulin resistance, at least during adolescence.

Differential transcriptomic profiles between omVAT and SAT depots. The use of both RNA-Seq and real-time PCR allowed further understanding of the interdepot differences at the molecular level by assessing potential differences in key pathways essential to regulation of lipolysis/adipogenesis, browning/beiging, and inflammation.

Although no significant differences between the 2 depots emerged regarding the regulatory genes for lipolysis and adipogenesis, we found differences in the phosphorylation of ATGL in omVAT compared with SAT, consistent with greater lipolytic activity of the omVAT depot (50-52), even at this stage. 

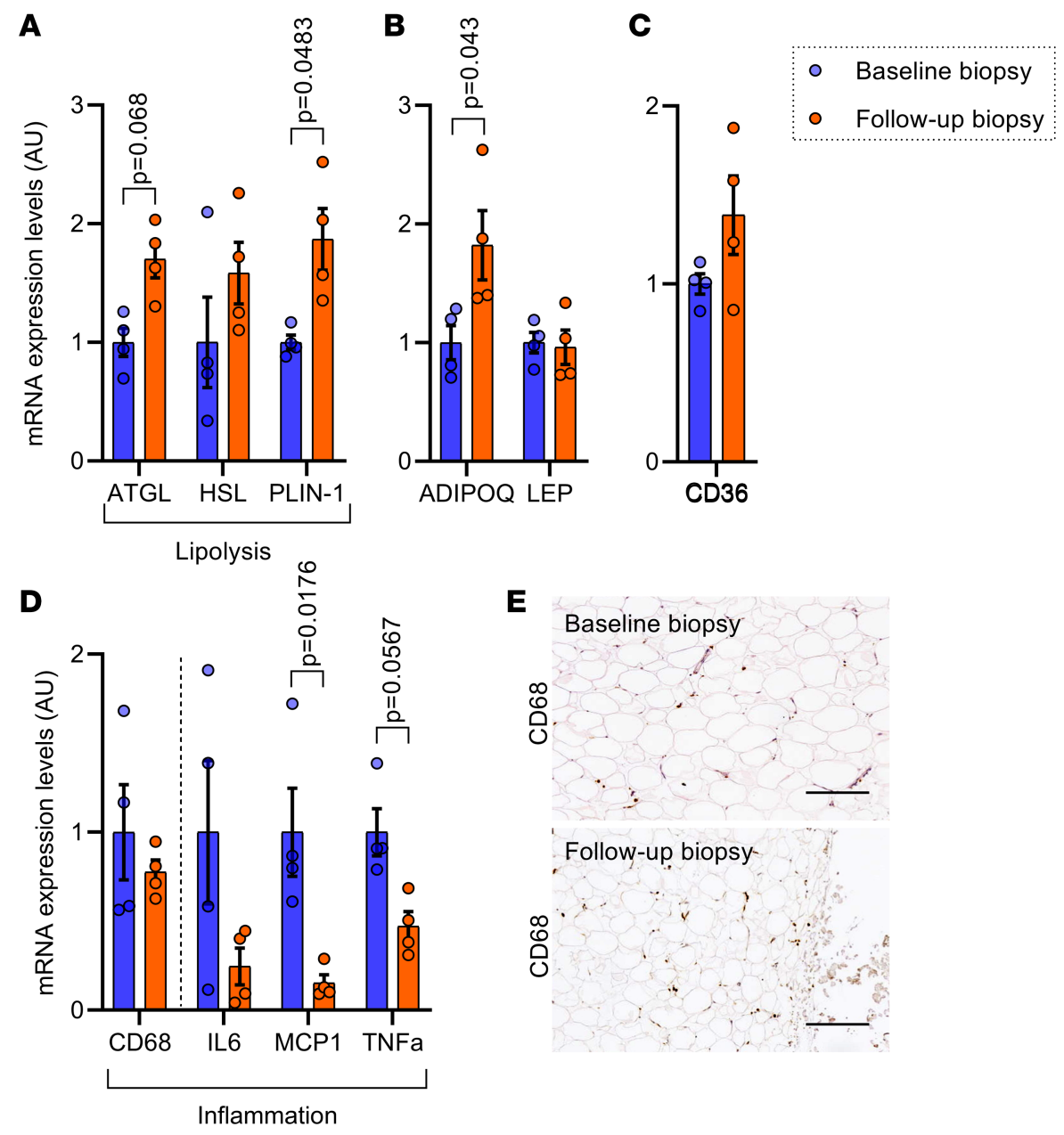

E
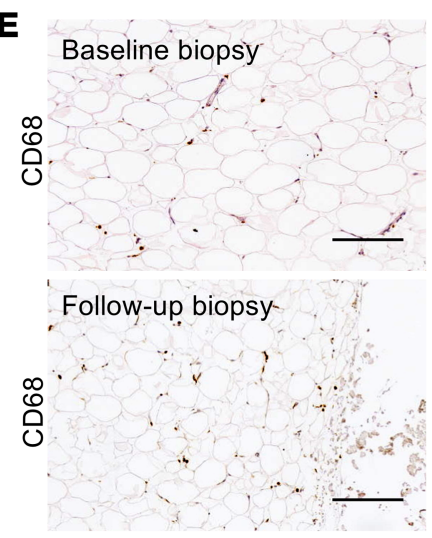

Figure 7. Variation in the expression of genes associated with lipid metabolism, adipogenesis, and inflammation in SAT after weight loss. Validation by real-time PCR of RNA-Seq results of a panel of genes involved in (A) lipolysis, (B and C) adipogenesis, and $(D)$ inflammation $(n=4)$. (E) Representative images of $C D 68^{+}$macrophage IHC staining showing the mRNA expression levels in SAT at baseline and after weight loss. Scale bar: $200 \mu \mathrm{m}$. Error bars represent mean \pm SEM. Gene expression data were compared using paired Student's $t$ test. $P$ values less than 0.05 were considered significant.

Despite the general thinking that the amount of brown adipose tissue (BAT) declines right after birth, several studies demonstrated the presence of brown-like adipocytes in white adipose tissue (WAT) also in kids and adults (53-55). Besides BAT, white adipocytes are also reported to be able to "convert" to brownlike cells in response to appropriate stimuli, such as different hormones and cold exposure (56, 57). However, the depot-specific expression of browning genes in human WAT is still poorly investigated. Therefore, of particular interest are the differences in the genes regulating the browning/beiging and inflammation in these 2 depots (see Figure 4). First, we found that the markers of genes regulating the browning of WAT were significantly upregulated in omVAT compared with SAT (Figure 4, A-C).

In accordance with our data, other studies showed that in adults omVAT did exhibit a distinct increase in UCP1 gene expression compared with SAT $(58,59)$. However, gene expression of other browning/beiging markers in human WAT obtained from adolescents with $\mathrm{SO}$ has not yet been described.

Moreover, the higher expression of $P G C 1 A, T B X 1$, and EBF2 was found to be related inversely and strongly to the omVAT cell diameter. In other words, the larger the cells in omVAT, the lower the level of gene expression (Figure 4). These findings would suggest that there might be a relation between cell size in omVAT and the activity of browning in this depot. At this point, however, this statement is purely speculative because we have no data on whether the activation of the browning process in the omVAT depot was present, as it was not assessed.

In contrast, in SAT, we found no evidence at baseline for the browning/beiging markers.

As for the genes regulating inflammation, we found that $C D 68$ as well as many of the proinflammatory genes were upregulated in SAT as opposed to omVAT. These differences were seen by RNA-Seq 


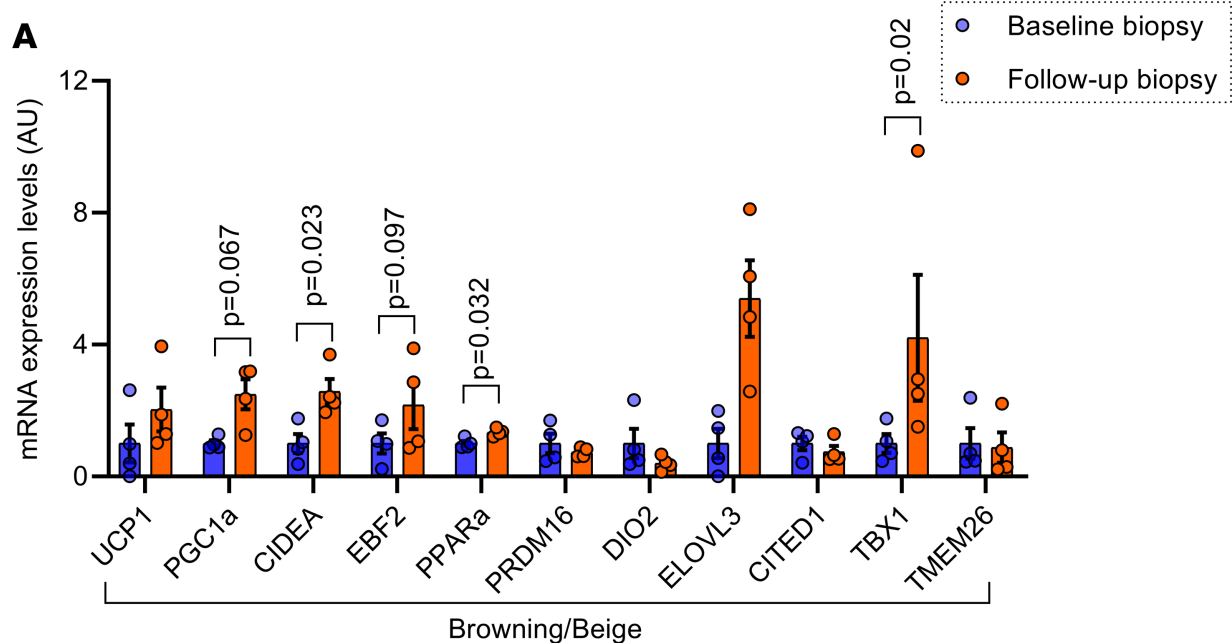

B

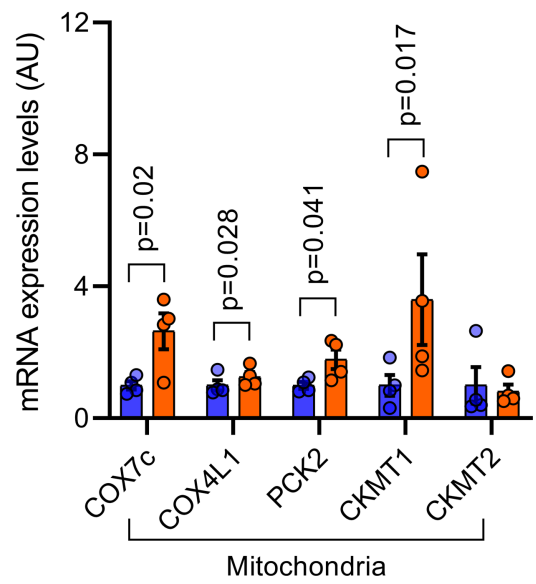

C Baseline biopsy
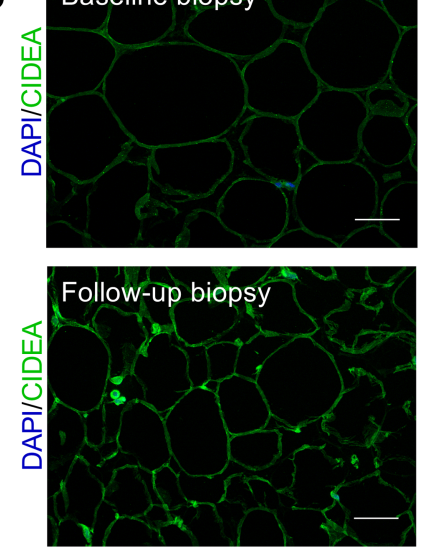

Figure 8. Upregulation of browning genes in SAT after weight loss. Validation by real-time PCR of RNA-Seq results of a panel of genes involved in (A) browning of white adipose tissue and (B) mitochondrial markers $(n=4)$. (C) Representative immunofluorescence staining shows an increase of CIDEA in SAT adipose tissue after weight loss (follow-up biopsy) compared with the baseline biopsy. Scale bar: $50 \mu \mathrm{m}$. Error bars represent mean \pm SEM. Gene expression data were compared using paired Student's $t$ test. $P$ values less than 0.05 were considered significant.

and confirmed by RT-PCR. Consistent with studies from Leibel et al. in obese adolescents (60), our data showed a similar trend but more robust reduction of $C D 68$ and other proinflammatory markers in the SAT depot. However, these data are in contrast with those reported in adults (49). Several papers reported that in a population of obese adults, visceral adipose tissue exhibits an evident increase of infiltrating macrophages as well as an elevated expression and secretion of proinflammatory cytokines (such as IL6 and MCP1) compared with SAT $(49,61-64)$. These differences in omVAT/SAT inflammation profiles between obese youths and adults support the idea that adolescence and adulthood are 2 metabolically different stages of human life.

Effects of SG-induced weight loss on cell size and gene expressions from SAT. All subjects lost a substantial amount of fat mass after the first 6 months following SG. Thereafter, the weight remained stable until the end of the study. As expected, insulin sensitivity also improved significantly in parallel with resolution of prediabetes and T2D. These remarkable clinical and metabolic improvements are very similar to those described in the young subjects from the Teen-LABS $(18,65)$.

The novelty of the current study relies on the mechanisms causing the observed changes in metabolism. First, we found a profound change in the cell size of the SAT of these girls after weight loss. Second, the lipolytic activity as measured by Western blotting showed a decrease in ATGL phosphorylation but an increase in PLIN1 phosphorylation. At baseline, PLIN1 was expressed and phosphorylated at very low levels in SAT adipocytes. Therefore, the lipolysis was regulated almost exclusively by ATGL phosphorylation. 
A

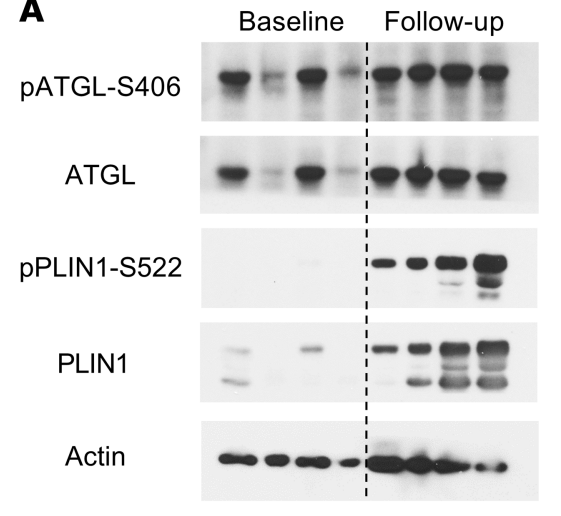

B

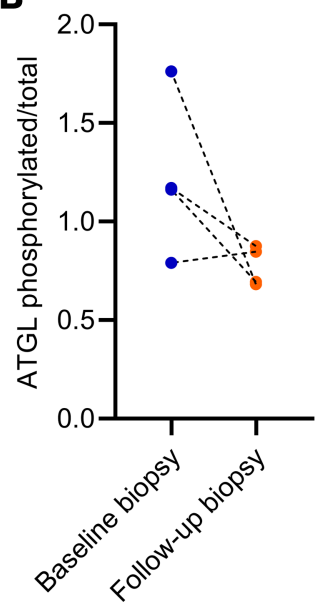

C

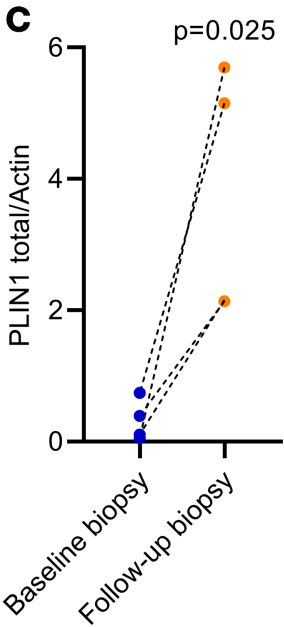

Figure 9. Switch of lipolytic activity toward activation of PLIN1 phosphorylation in SAT after weight loss. (A) Protein expression of both total and phosphorylated ATCL and PLIN1 in SAT before (baseline) and after weight loss (follow-up) $(n=4)$. (B) Quantification of ATCL phosphorylated/total ratio at baseline and follow-up biopsy $(n=4)$. (C) Quantification of PLIN1 total/actin ratio at baseline and follow-up biopsy $(n=4)$. Data were compared using paired Student's $t$ test. $P$ values less than 0.05 were considered significant.

On the contrary, after weight loss, PLIN1 was reexpressed and phosphorylated. It was therefore able to lead lipolysis in combination with ATGL (which almost did not change in phosphorylation level after weight loss) (see Figure 9). The increase in total PLIN1 expression after weight loss that we observed in our cohort was in accordance with previous observations in SAT obtained from adults (64, 66-68).

Perhaps the most novel findings are the induction of the gene markers related to browning/beiging of WAT (see Figure 8) and the downregulation of the inflammatory genes in SAT after weight loss (see Figure 7).

The downregulation of inflammatory genes in SAT after weight loss that we observed in our cohort of adolescent girls is in accordance with the observation of other groups that showed a reduction in inflammatory markers, such as $C D 68$ and $M C P 1$, after surgery or after caloric restriction $(65,69-71)$. In contrast, others described a reduction or even no significant changes in $I L 6, T N F$, or $I L 18$ after weight loss $(72,73)$.

Similar disagreement was observed in relation to the overexpression of gene markers of the browning of WAT in SAT induced after weight loss. In fact, although some groups confirmed the presence of beige adipocytes in SAT after weight loss (74), others showed a reduction in key genes associated with the browning process (70).

A number of studies in mice have shown that activation of browning/beiging of WAT facilitates weight loss, ameliorates insulin resistance, and corrects hyperlipidemia in the obese state (75-78). Other studies in mice have demonstrated that browning/beiging is induced by caloric restriction, mediated mainly through IL-4 and other M2-polarized macrophages' cytokine signaling (79-81). It is also important to highlight that Zuriaga et al. clearly describe an inverse pattern of browning gene expression between mice and humans, suggesting that extrapolation of data from mice on adipose biology to humans should be done with some caution (82). Consistent with other clinical studies done in human adults, our findings showed an increase of browning/beiging markers in omVAT that led to an improvement of oxidative metabolism and decrease of body fat mass $(75,82,83)$. However, in our cohort, the browning activation happened at a much younger age. These opposite changes in the regulatory browning genes and inflammatory genes would suggest modulatory effects on the changes in insulin sensitivity.

Our finding showing the induction of browning/beiging in SAT after SG-induced weight loss is in contrast to findings reported in adults after caloric restriction (84). A recent study in a large cohort of adults who were obese showed that caloric restriction and diet-induced weight loss diminishes browning features in SAT and that diet-induced changes in body fat are independent of subcutaneous abdominal WAT browning (84). The result and conclusion of the lack of activation of browning in the SAT depot induced by diet-induced weight loss cannot be extrapolated to bariatric surgery-induced weight loss. Indeed, several studies recently reported bariatric surgery-induced BAT activation and WAT browning in the neck (85-88), 
White Adipose Tissue Baseline

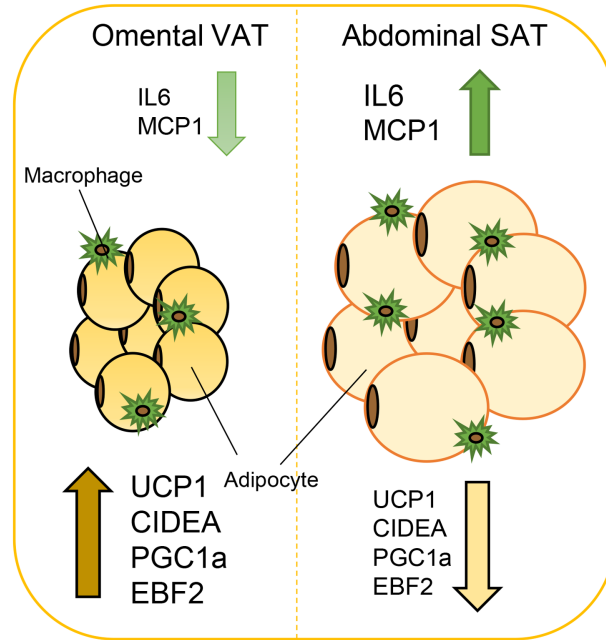

High Insulin-Resistance (IR)

\section{White Adipose Tissue After weight loss}

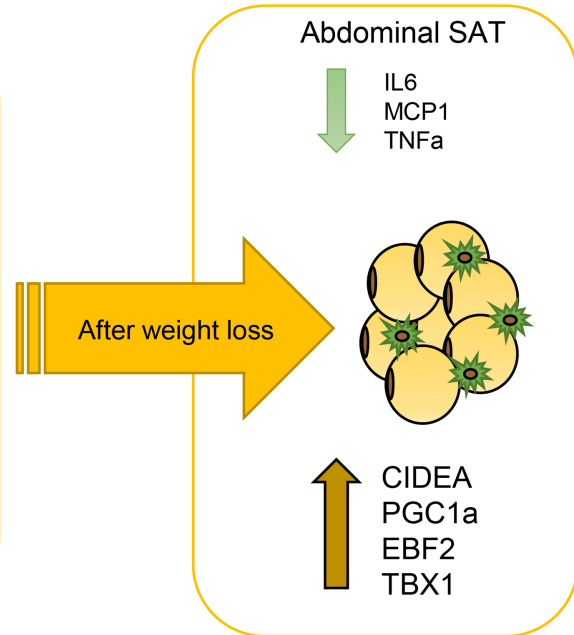

Reduction in IR

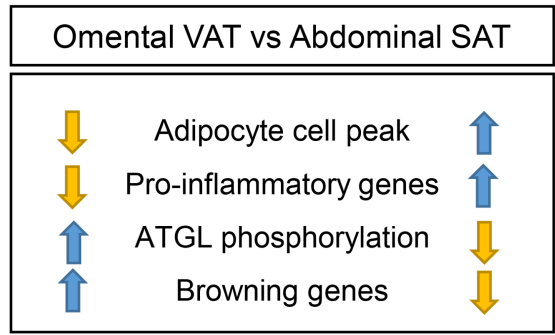

SAT baseline vs SAT after weight loss

Figure 10. Summary of the key study findings at baseline before the bariatric surgery and after weight loss.

suggesting perhaps that activation of WAT browning may be among one of the many potential mechanisms by which bariatric surgery causes weight loss.

Our study limitations are due to the small number of subjects undergoing SG and consenting to have a repeated follow-up biopsy. Moreover, the limited amount of tissue collected during the biopsies limited our ability to perform further functional assays/experiments to assess browning activity and in vivo/vitro lipolysis assays. We were able to measure only the mRNA expression of the pro-/antiinflammatory markers in this study, which could be different from protein secretion, although in the literature it was suggested that mRNA of adiponectin and IL-6 correlates well with the protein secretion (89-92). Furthermore, in our study, we did not have paired omental SAT biopsies from a non-caloric-restricted group. Also, we did not have a non-surgically treated group (weight loss induced by caloric restriction only) to identify whether the observed metabolic changes were due to caloric restriction or SG.

In conclusion, this study demonstrates that in adolescent girls with SO there are radical differences in the cellular and transcriptomic profiles of paired biopsy samples from the omVAT and SAT depots. We were able to identify in the omVAT a specific profile consisting of the increase of browning/beiging markers and decrease of proinflammatory markers. Of note, following weight loss, the SAT depot cellular and transcriptomic profile changed profoundly and was associated with a reversal in insulin resistance.

\section{Methods}

Study design and subject characteristics. From The Yale Study of Body Fat Patterning in Obese Adolescents, we recruited 10 girls with class $2 / 3$ obesity (7) who elected to have bariatric surgery as a weight loss intervention. These 10 obese female patients were between 16 and 22 years old ( 7 adolescents and 3 young adults), underwent SG, and agreed to have a paired subcutaneous periumbilical (SAT) and visceral-omental (omVAT) adipose tissue biopsy on the same day of the surgery procedure at Yale done by our pediatric bariatric surgeon. Body weight and metabolic/clinical parameters were monitored during follow-up visits 
in 9 patients; 1 patient left the study/follow-up after surgery for personal reasons. Four patients consented to have a second SAT biopsy after weight stabilization following weight loss. The follow-up biopsies were performed at Yale Center for Clinical Investigation-Hospital Research Unit (YCCI-HRU) after administration of local anesthesia (lidocaine without adrenaline/epinephrine).

The clinical characteristics of enrolled subjects are described in Table 1.

Analytical methods. Plasma glucose levels were measured using the Yellow Springs Instruments 2700 STAT Analyzer, and lipid levels were measured using an autoanalyzer (747-200; Roche-Hitachi). Plasma insulin, adiponectin, and leptin levels were measured using radioimmunoassay (Linco).

Adipocyte size measurement. Samples of abdominal subcutaneous and visceral-omental adipose tissue were collected during SG surgery. Two 20- to 30-mg samples were immediately used for adipose cell size distribution analysis by osmium tetroxide fixation (Multisizer 4; Beckman Coulter). We performed a curve-fitting analysis technique as previously described (27). We calculated the "peak diameter" of the large adipocytes, defined as the mean diameter that showed the highest frequency of the large cell population. In addition to determining the peak diameter of the large adipose cells as described, we calculated the "\% of adipose cells above" (percentage of large cells) and "\% below" (percentage of small cells) the nadir.

Real-time PCR. Fat tissues were homogenized into QIAzol Lysis Reagent (QIAGEN Inc.). Total RNA was isolated using RNeasy Mini Kit (QIAGEN Inc.) and reverse-transcribed to cDNA using High-Capacity cDNA Reverse Transcription Kit (Applied Biosystems, Thermo Fisher Scientific). Real-time PCR was performed using SYBR Green master mix (Bio-Rad) on an Applied Biosystems 7500 Fast Real-Time PCR System (Thermo Fisher Scientific). The expression of each gene was normalized to the expression of the housekeeping gene TATA-binding protein. All reactions were performed in triplicate. The relative expression levels of each transcript were calculated using the $2^{-\Delta \Delta C t}$ method and values were expressed in AU. The list of all primers used is in Supplemental Table 1.

RNA next-generation sequencing and analysis. The total RNA isolated from SAT and omVAT depots was measured using an Agilent 2200 Bioanalyzer to evaluate quality and quantity. RNAs with RNA integrity number greater than 8.0 were used to construct the cDNA library, and sequencing was subsequently performed with Illumina HiSeq 4000.

The sequencing reads for each of the samples were aligned to the GRCh38 human reference genome using HISAT2 (93). Then, gene-level read counts were generated using the featureCounts function of Rsubread (94), based on annotations from the ENCODE v27 GTF file. Differential gene expression was performed using DESeq2 (95). The DESeq2 analysis results were submitted to the IPA software (QIAGEN Inc.) (96), and a core analysis was used to perform pathway enrichment analysis on the differentially expressed genes. RNA-Seq data will be deposited into the National Center for Biotechnology Information's Gene Expression Omnibus, accession GSE145284.

Western blotting. Protein extraction from fat tissues was performed using RIPA buffer supplemented with phosphatase (PhosSTOP, Roche) and proteinase (cOmplete MINI, Roche) inhibitors, and protein content was quantified using BCA assay (Pierce, Thermo Fisher Scientific). After denaturation with heat and $\beta$-mercaptoethanol, an equal amount of proteins was run in $4 \%-12 \%$ Tris-Glycine Gel (Novex, Invitrogen, Thermo Fisher Scientific). Proteins were transferred to PVDF membranes (MilliporeSigma) by semidry transfer and blocked with 3\% BSA. Membranes were blotted overnight at $4^{\circ} \mathrm{C}$ with specific primary antibodies. Actin (catalog 4967), ATGL (catalog 2138), HSL (catalog 4107), p-Ser660 HSL (catalog 4126), and perilipin 1 (catalog 3467) antibodies were from Cell Signaling Technology; p-Ser406 ATGL (catalog ab135093) antibody was from Abcam, and p-Ser522 perilipin 1 (catalog 4856) antibody was from VALA Sciences. Membranes were washed in TBS-Tween (TBS-T) 3 times and then incubated 1 hour with HRP-conjugated secondary antibody (catalog 7076 and catalog 7074, Cell Signaling Technology). After 3 washings in TBS-T, the specific band was visualized using enhanced ECL chemiluminescence substrate (Pierce, Thermo Fisher Scientific). Films were developed within the linear dynamic range of signal intensity and then scanned. The intensity of the bands was measured using ImageJ software (NIH).

Because of the limited amount of SAT tissue collected, the protein expression assay was performed on $n=8$ SAT tissues collected during the SG.

Immunofluorescence and IHC. Adipose tissue biopsy samples were used for immunofluorescence staining for browning markers (UCP-1, R\&D Systems, Bio-Techne MAB6158; CIDEA-FITC, Bioss bs-7649R). Formalin-fixed and paraffin-embedded tissue blocks were deparaffinized and rehydrated. Sections were rinsed in PBS before epitope retrieval with $10 \mathrm{mM}$ citrate buffer ( $\mathrm{pH}$ 6.0). After blocking in 5\% BSA at 
room temperature, sections were incubated overnight at $4^{\circ} \mathrm{C}$ with primary antibodies. After rinsing in PBSTween, sections were incubated with specific fluorescent secondary antibodies conjugated to Alexa Fluor 488 (catalog A-11001, Invitrogen, Thermo Fisher Scientific) and mounted with Prolong Antifade mounting medium with DAPI (Invitrogen, Thermo Fisher Scientific). Sections stained with secondary antibody while omitting the primary antibody served as negative controls. Images were acquired using a Leica SP5 confocal microscope using the following settings: blue fluorescence: 405 laser power $=16 \%$, gain $=1041$; green fluorescence: 488 laser power $=20 \%$, gain $=30$; all images were acquired using the same pixel dwell time settings $=721 \mathrm{~ns}$.

IHC staining was performed using a standard protocol on sections from formalin-fixed, paraffin-embedded tissue blocks. Briefly, sections were deparaffinized, rehydrated, and treated with $10 \mathrm{mmol} / \mathrm{L}$ citrate buffer ( $\mathrm{pH}$ 6.0) in a steamer, and then endogenous peroxidase was blocked with $3 \% \mathrm{H}_{2} \mathrm{O}_{2}$. The sections were then incubated for 1 hour at room temperature with primary antibodies, mouse monoclonal antiCD68 (Ab-3 clone KP1; Thermo Fisher Scientific). After rinsing in Tris-buffered saline solution containing $0.25 \%$ Triton X-100 ( $\mathrm{pH} 7.2$ ), sections were incubated with ENVISION+ (K4007 or K4011; DAKO), followed by visualization with 3.39-diaminobenzidine tetrachloride (DAKO). All sections were counterstained with GILL III hematoxylin, dehydrated, and coverslipped with a resinous mounting medium. Images were acquired using Aperio digital whole-slide scanner and representative images were presented.

Statistics. Before analysis, the data were tested for normality. When appropriate, log-transformed data were tested with 2-tailed Student's $t$ test. Weighted means for adipocytes were calculated for adipose cell size of the abdominal and visceral fat. Wilcoxon's matched-pairs signed-rank test was applied, unless otherwise specified. Correlation tests were performed by linear regression test and by nonparametric Spearman's correlation analysis. For all analyses a $P$ value less than 0.05 was considered statistically significant. Data are expressed as mean \pm SEM. GraphPad Prism 8.2 was used for all statistical analysis.

Study approval. The nature and potential risks of the study were explained to all subjects before investigators obtained subjects' written informed consent. The study was approved by the ethics committees of Yale University Hospital (HIC1109009034 and HIC1503015459) and registered on ClinicalTrials.gov (NCT02004561 and NTC02395003, respectively).

\section{Author contributions}

ET performed all experiments and data analyses and wrote the manuscript. JN processed the tissues and performed cell size measurements. MVN and RG recruited the subjects. BP obtained informed consent. GSN performed the gastric sleeve surgery and provided the fat tissue biopsies. AVM collected the follow-up biopsies. JRK performed the RNA-Seq analysis and helped with the interpretation of the analysis. GIS provided laboratory space and helped with the setup of the methods used. SC designed the study and wrote the manuscript. All authors contributed to the interpretation of the data. SC is the guarantor of this work, had full access to all the data in the study, and takes responsibility for the integrity of the data and the accuracy of the data analysis.

\section{Acknowledgments}

The authors thank all the volunteers and research nurses at the Yale HRU for their skillful help in the study.

This study was supported by the Robert E. Leet and Clara Guthrie Patterson Trust (Mentored Research Award to JN); NIH Eunice Kennedy Shriver National Institute of Child Health and Human Development grants R01-HD-40787, R01-HD-28016, and K24-HD-01464 to SC; Clinical and Translational Science Award grant UL1-TR001863 from the National Center for Advancing Translational Science, a component of the NIH; grant R01-EB006494 (Bioimage Suite); and Distinguished Clinical Scientist Award from the American Diabetes Association (to SC), as well as grants DK-49230 and DK-085638 (to GIS) and the Diabetes Research Center grant P30-DK-045735.

JN's present address is: Section of Pulmonary, Critical Care, and Sleep Medicine, Department of Internal Medicine, Yale University School of Medicine, New Haven, Connecticut, USA.

Address correspondence to: Sonia Caprio, Yale School of Medicine, 330 Cedar Street, LMP 3085, New Haven, Connecticut 06510, USA. Phone: 203.785.5692; Email: sonia.caprio@yale.edu. 
1. Kelly AS, et al. Severe obesity in children and adolescents: identification, associated health risks, and treatment approaches: a scientific statement from the American Heart Association. Circulation. 2013;128(15):1689-1712.

2. Skelton JA, Cook SR, Auinger P, Klein JD, Barlow SE. Prevalence and trends of severe obesity among US children and adolescents. Acad Pediatr. 2009;9(5):322-329.

3. Claire Wang Y, Gortmaker SL, Taveras EM. Trends and racial/ethnic disparities in severe obesity among US children and adolescents, 1976-2006. Int J Pediatr Obes. 2011;6(1):12-20.

4. Flegal KM, Wei R, Ogden CL, Freedman DS, Johnson CL, Curtin LR. Characterizing extreme values of body mass index-forage by using the 2000 Centers for Disease Control and Prevention growth charts. Am J Clin Nutr. 2009;90(5):1314-1320.

5. Freedman DS, Mei Z, Srinivasan SR, Berenson GS, Dietz WH. Cardiovascular risk factors and excess adiposity among overweight children and adolescents: the Bogalusa Heart Study. J Pediatr. 2007;150(1):12-17.e2.

6. Koebnick C, et al. Prevalence of extreme obesity in a multiethnic cohort of children and adolescents. J Pediatr. 2010;157(1):26-31.e2

7. Skinner AC, Skelton JA. Prevalence and trends in obesity and severe obesity among children in the United States, 1999-2012. JAMA Pediatr. 2014;168(6):561-566.

8. Baker JL, Olsen LW, Sørensen TI. Childhood body-mass index and the risk of coronary heart disease in adulthood. $N$ Engl $J$ Med. 2007;357(23):2329-2337.

9. Franks PW, Hanson RL, Knowler WC, Sievers ML, Bennett PH, Looker HC. Childhood obesity, other cardiovascular risk factors, and premature death. N Engl J Med. 2010;362(6):485-493.

10. Juonala M, et al. Childhood adiposity, adult adiposity, and cardiovascular risk factors. $N$ Engl J Med. 2011;365(20):1876-1885

11. Ma J, Flanders WD, Ward EM, Jemal A. Body mass index in young adulthood and premature death: analyses of the US National Health Interview Survey linked mortality files. Am J Epidemiol. 2011;174(8):934-944.

12. Morrison JA, Friedman LA, Gray-McGuire C. Metabolic syndrome in childhood predicts adult cardiovascular disease 25 years later: the Princeton Lipid Research Clinics Follow-up Study. Pediatrics. 2007;120(2):340-345.

13. Must A, Jacques PF, Dallal GE, Bajema CJ, Dietz WH. Long-term morbidity and mortality of overweight adolescents. A follow-up of the Harvard Growth Study of 1922 to 1935. N Engl J Med. 1992;327(19):1350-5.

14. Owen CG, et al. Is body mass index before middle age related to coronary heart disease risk in later life? Evidence from observational studies. Int J Obes (Lond). 2009;33(8):866-877.

15. Park MH, Falconer C, Viner RM, Kinra S. The impact of childhood obesity on morbidity and mortality in adulthood: a systematic review. Obes Rev. 2012;13(11):985-1000.

16. Tirosh A, et al. Adolescent BMI trajectory and risk of diabetes versus coronary disease. NEngl J Med. 2011;364(14):1315-1325.

17. Danielsson P, Kowalski J, Ekblom Ö, Marcus C. Response of severely obese children and adolescents to behavioral treatment. Arch Pediatr Adolesc Med. 2012;166(12):1103-1108.

18. Inge TH, et al. Five-year outcomes of gastric bypass in adolescents as compared with adults. NEngl J Med. 2019;380(22):2136-2145.

19. Styne DM, et al. Pediatric obesity-assessment, treatment, and prevention: an Endocrine Society Clinical Practice Guideline. J Clin Endocrinol Metab. 2017;102(3):709-757.

20. Zimmermann R, et al. Fat mobilization in adipose tissue is promoted by adipose triglyceride lipase. Science. 2004;306(5700):1383-1386.

21. Jenkins CM, Mancuso DJ, Yan W, Sims HF, Gibson B, Gross RW. Identification, cloning, expression, and purification of three novel human calcium-independent phospholipase A2 family members possessing triacylglycerol lipase and acylglycerol transacylase activities. J Biol Chem. 2004;279(47):48968-48975.

22. Villena JA, Roy S, Sarkadi-Nagy E, Kim KH, Sul HS. Desnutrin, an adipocyte gene encoding a novel patatin domain-contain ing protein, is induced by fasting and glucocorticoids: ectopic expression of desnutrin increases triglyceride hydrolysis. $J$ Biol Chem. 2004;279(45):47066-47075.

23. Lass A, et al. Adipose triglyceride lipase-mediated lipolysis of cellular fat stores is activated by CGI-58 and defective in Chanarin-Dorfman Syndrome. Cell Metab. 2006;3(5):309-319.

24. Itabe H, Yamaguchi T, Nimura S, Sasabe N. Perilipins: a diversity of intracellular lipid droplet proteins. Lipids Health Dis. 2017;16(1):83

25. Kraemer FB, Shen WJ. Hormone-sensitive lipase: control of intracellular tri-(di-)acylglycerol and cholesteryl ester hydrolysis. J Lipid Res. 2002;43(10):1585-1594.

26. Tornqvist H, Belfrage P. Monoacylglycerol lipase from rat adipose tissue. Meth Enzymol. 1981;71 pt C:646-652.

27. Kursawe R, et al. Cellularity and adipogenic profile of the abdominal subcutaneous adipose tissue from obese adolescents: association with insulin resistance and hepatic steatosis. Diabetes. 2010;59(9):2288-2296.

28. Kajimura S, et al. Initiation of myoblast to brown fat switch by a PRDM16-C/EBP-beta transcriptional complex. Nature. 2009;460(7259):1154-1158.

29. Ito M, Nagasawa M, Hara T, Ide T, Murakami K. Differential roles of CIDEA and CIDEC in insulin-induced anti-apoptosis and lipid droplet formation in human adipocytes. J Lipid Res. 2010;51(7):1676-1684.

30. Abreu-Vieira G, et al. Cidea improves the metabolic profile through expansion of adipose tissue. Nat Commun. 2015;6:7433.

31. Sharp LZ, et al. Human BAT possesses molecular signatures that resemble beige/brite cells. PLoS ONE. 2012;7(11):e49452.

32. Wu J, et al. Beige adipocytes are a distinct type of thermogenic fat cell in mouse and human. Cell. 2012;150(2):366-376.

33. Kazak L, et al. A creatine-driven substrate cycle enhances energy expenditure and thermogenesis in beige fat. Cell. 2015;163(3):643-655.

34. Rosenwald M, Wolfrum C. The origin and definition of brite versus white and classical brown adipocytes. Adipocyte. 2014;3(1):4-9.

35. Huttunen P, Hirvonen J, Kinnula V. The occurrence of brown adipose tissue in outdoor workers. Eur J Appl Physiol Occup Physiol. 1981;46(4):339-345.

36. Lee $\mathrm{P}$, et al. Temperature-acclimated brown adipose tissue modulates insulin sensitivity in humans. Diabetes. 2014;63(11):3686-3698.

37. van der Lans AA, et al. Cold acclimation recruits human brown fat and increases nonshivering thermogenesis. J Clin Invest. 2013;123(8):3395-3403

38. Yoneshiro T, et al. Recruited brown adipose tissue as an antiobesity agent in humans. J Clin Invest. 2013;123(8):3404-3408 
39. Lowell BB, Spiegelman BM. Towards a molecular understanding of adaptive thermogenesis. Nature. 2000;404(6778):652-660.

40. Kern PA, et al. The effects of temperature and seasons on subcutaneous white adipose tissue in humans: evidence for thermogenic gene induction. J Clin Endocrinol Metab. 2014;99(12):E2772-E2779.

41. Dietz WH. Periods of risk in childhood for the development of adult obesity--what do we need to learn? J Nutr. 1997;127(9):1884S-1886S

42. Lee MJ, Fried SK. Sex-dependent depot differences in adipose tissue development and function; role of sex steroids. J Obes Metab Syndr. 2017;26(3):172-180.

43. Karastergiou K, Fried SK. Cellular mechanisms driving sex differences in adipose tissue biology and body shape in humans and mouse models. Adv Exp Med Biol. 2017;1043:29-51.

44. Karastergiou K, Smith SR, Greenberg AS, Fried SK. Sex differences in human adipose tissues - the biology of pear shape. Biol Sex Differ. 2012;3(1):13.

45. Fried SK, Lee MJ, Karastergiou K. Shaping fat distribution: New insights into the molecular determinants of depot- and sex-dependent adipose biology. Obesity (Silver Spring). 2015;23(7):1345-1352.

46. Knittle JL, Timmers K, Ginsberg-Fellner F, Brown RE, Katz DP. The growth of adipose tissue in children and adolescents. Cross-sectional and longitudinal studies of adipose cell number and size. J Clin Invest. 1979;63(2):239-246.

47. Spalding KL, et al. Dynamics of fat cell turnover in humans. Nature. 2008;453(7196):783-787.

48. Landgraf $\mathrm{K}$, et al. Evidence of early alterations in adipose tissue biology and function and its association with obesity-related inflammation and insulin resistance in children. Diabetes. 2015;64(4):1249-1261.

49. Liu A, et al. Differential intra-abdominal adipose tissue profiling in obese, insulin-resistant women. Obes Surg. 2009;19(11):1564-1573.

50. Richelsen B, Pedersen SB, Møller-Pedersen T, Bak JF. Regional differences in triglyceride breakdown in human adipose tissue: effects of catecholamines, insulin, and prostaglandin E2. Metab Clin Exp. 1991;40(9):990-996.

51. Petrus $P$, et al. Depot-specific differences in fatty acid composition and distinct associations with lipogenic gene expression in abdominal adipose tissue of obese women. Int J Obes (Lond). 2017;41(8):1295-1298.

52. Wajchenberg BL. Subcutaneous and visceral adipose tissue: their relation to the metabolic syndrome. Endocr Rev. 2000;21(6):697-738.

53. Rockstroh D, et al. Direct evidence of brown adipocytes in different fat depots in children. PLoS ONE. 2015;10(2):e0117841

54. Cypess AM, et al. Identification and importance of brown adipose tissue in adult humans. NEngl J Med. 2009;360(15):1509-1517.

55. Nedergaard J, Bengtsson T, Cannon B. Unexpected evidence for active brown adipose tissue in adult humans. Am J Physiol Endocrinol Metab. 2007;293(2):E444-E452.

56. Bartelt A, Heeren J. Adipose tissue browning and metabolic health. Nat Rev Endocrinol. 2014;10(1):24-36.

57. Lo KA, Sun L. Turning WAT into BAT: a review on regulators controlling the browning of white adipocytes. Biosci Rep. 2013;33(5):e00065.

58. Lim J, et al. Depot-specific UCP1 expression in human white adipose tissue and its association with obesity-related markers. Int J Obes (Lond). 2020;44(3):697-706.

59. Bettini S, et al. Resting energy expenditure, insulin resistance and UCP1 expression in human subcutaneous and visceral adipose tissue of patients with obesity. Front Endocrinol (Lausanne). 2019;10:548.

60. Zhang Y, et al. Fat cell size and adipokine expression in relation to gender, depot, and metabolic risk factors in morbidly obese adolescents. Obesity (Silver Spring). 2014;22(3):691-697.

61. Harman-Boehm I, et al. Macrophage infiltration into omental versus subcutaneous fat across different populations: effect of regional adiposity and the comorbidities of obesity. J Clin Endocrinol Metab. 2007;92(6):2240-2247.

62. Fried SK, Bunkin DA, Greenberg AS. Omental and subcutaneous adipose tissues of obese subjects release interleukin-6: depot difference and regulation by glucocorticoid. J Clin Endocrinol Metab. 1998;83(3):847-850.

63. Bruun JM, Lihn AS, Pedersen SB, Richelsen B. Monocyte chemoattractant protein-1 release is higher in visceral than subcutaneous human adipose tissue (AT): implication of macrophages resident in the AT. J Clin Endocrinol Metab. 2005;90(4):2282-2289.

64. Moreno-Navarrete JM, et al. CIDEC/FSP27 and PLIN1 gene expression run in parallel to mitochondrial genes in human adipose tissue, both increasing after weight loss. Int J Obes (Lond). 2014;38(6):865-872.

65. Kelly AS, Ryder JR, Marlatt KL, Rudser KD, Jenkins T, Inge TH. Changes in inflammation, oxidative stress and adipokines following bariatric surgery among adolescents with severe obesity. Int J Obes (Lond). 2016;40(2):275-280.

66. Cortes de Oliveira C, et al. Influence of expression of UCP3, PLIN1 and PPARG2 on the oxidation of substrates after hypocaloric dietary intervention. Clin Nutr. 2018;37(4):1383-1388.

67. Wang Y, et al. Perilipin expression in human adipose tissues: effects of severe obesity, gender, and depot. Obes Res. 2003;11(8):930-936.

68. Mottagui-Tabar S, et al. Evidence for an important role of perilipin in the regulation of human adipocyte lipolysis. Diabetologia. 2003;46(6):789-797.

69. Cancello R, et al. Reduction of macrophage infiltration and chemoattractant gene expression changes in white adipose tissue of morbidly obese subjects after surgery-induced weight loss. Diabetes. 2005;54(8):2277-2286.

70. Jürets A, et al. Upregulated TNF expression 1 year after bariatric surgery reflects a cachexia-like state in subcutaneous adipose tissue. Obes Surg. 2017;27(6):1514-1523.

71. Clément $\mathrm{K}$, et al. Weight loss regulates inflammation-related genes in white adipose tissue of obese subjects. FASEB J. 2004;18(14):1657-1669.

72. Katsogiannos P, et al. Early changes in adipose tissue morphology, gene expression, and metabolism after RYGB in patients with obesity and T2D. J Clin Endocrinol Metab. 2019;104(7):2601-2613.

73. Magkos F, et al. Effects of moderate and subsequent progressive weight loss on metabolic function and adipose tissue biology in humans with obesity. Cell Metab. 2016;23(4):591-601.

74. Mardinoglu A, et al. Extensive weight loss reveals distinct gene expression changes in human subcutaneous and visceral adipose tissue. Sci Rep. 2015;5:14841.

75. Xue Y, Xu X, Zhang XQ, Farokhzad OC, Langer R. Preventing diet-induced obesity in mice by adipose tissue transformation 
and angiogenesis using targeted nanoparticles. Proc Natl Acad Sci U S A. 2016;113(20):5552-5557.

76. Wang S, et al. Resveratrol induces brown-like adipocyte formation in white fat through activation of AMP-activated protein kinase (AMPK) $\alpha 1$. Int J Obes (Lond). 2015;39(6):967-976.

77. Liu X, et al. Brown adipose tissue transplantation reverses obesity in Ob/Ob mice. Endocrinology. 2015;156(7):2461-2469.

78. Abdullahi A, Jeschke MG. Taming the flames: targeting white adipose tissue browning in hypermetabolic conditions. Endocr Rev. 2017;38(6):538-549.

79. Fabbiano S, et al. Caloric restriction leads to browning of white adipose tissue through type 2 immune signaling. Cell Metab. 2016;24(3):434-446.

80. Nguyen $\mathrm{KD}$, et al. Alternatively activated macrophages produce catecholamines to sustain adaptive thermogenesis. Nature 2011;480(7375):104-108.

81. Rao RR, et al. Meteorin-like is a hormone that regulates immune-adipose interactions to increase beige fat thermogenesis. Cell. 2014;157(6):1279-1291.

82. Zuriaga MA, Fuster JJ, Gokce N, Walsh K. Humans and mice display opposing patterns of "browning" gene expression in visceral and subcutaneous white adipose tissue depots. Front Cardiovasc Med. 2017;4:27

83. Sacks H, Symonds ME. Anatomical locations of human brown adipose tissue: functional relevance and implications in obesity and type 2 diabetes. Diabetes. 2013;62(6):1783-1790.

84. Barquissau V, et al. Caloric restriction and diet-induced weight loss do not induce browning of human subcutaneous white adipose tissue in women and men with obesity. Cell Rep. 2018;22(4):1079-1089.

85. Neinast MD, et al. Activation of natriuretic peptides and the sympathetic nervous system following Roux-en-Y gastric bypass is associated with gonadal adipose tissues browning. Mol Metab. 2015;4(5):427-436.

86. Oliveira BA, et al. UCP1 and UCP3 expression is associated with lipid and carbohydrate oxidation and body composition. PLoS ONE. 2016;11(3):e0150811.

87. Rachid B, et al. Distinct regulation of hypothalamic and brown/beige adipose tissue activities in human obesity. Int J Obes (Lond). 2015;39(10):1515-1522.

88. Vijgen GH, et al. Increase in brown adipose tissue activity after weight loss in morbidly obese subjects. J Clin Endocrinol Metab. 2012;97(7):E1229-E1233

89. Hoffstedt J, Arvidsson E, Sjölin E, Wåhlén K, Arner P. Adipose tissue adiponectin production and adiponectin serum concentration in human obesity and insulin resistance. J Clin Endocrinol Metab. 2004;89(3):1391-1396.

90. Fisher FM, et al. Differences in adiponectin protein expression: effect of fat depots and type 2 diabetic status. Horm Metab Res. 2002;34(11-12):650-654

91. Fain JN, Bahouth SW, Madan AK. Involvement of multiple signaling pathways in the post-bariatric induction of IL-6 and IL-8 mRNA and release in human visceral adipose tissue. Biochem Pharmacol. 2005;69(9):1315-1324.

92. Arvidsson E, Blomqvist L, Rydén M. Depot-specific differences in perilipin mRNA but not protein expression in obesity. $J$ Intern Med. 2004;255(5):595-601.

93. Kim D, Langmead B, Salzberg SL. HISAT: a fast spliced aligner with low memory requirements. Nat Methods. 2015;12(4):357-360

94. Liao Y, Smyth GK, Shi W. The Subread aligner: fast, accurate and scalable read mapping by seed-and-vote. Nucleic Acids Res. 2013;41(10):e108.

95. Love MI, Huber W, Anders S. Moderated estimation of fold change and dispersion for RNA-seq data with DESeq2. Genome Biol. 2014;15(12):550.

96. Krämer A, Green G, Pollard J Jr, Tugendreich S. Causal analysis approaches in Ingenuity Pathway Analysis. Bioinformatics. 2014;30(4):523-530 